\title{
Mode competition in modulated Taylor-Couette flow
}

\author{
M. AVILA ${ }^{1}$, M. J. BELISLE ${ }^{2} \dagger$, J. M. LOPEZ $Z^{3}$, F. MARQUES \\ AND W. S. SAR I C \\ ${ }^{1}$ Departament de Física Aplicada, Univ. Politècnica de Catalunya, Barcelona 08034, Spain \\ ${ }^{2}$ Department of Mechanical and Aerospace Engineering, Arizona State University, Tempe, AZ 5287, \\ USA \\ ${ }^{3}$ Department of Mathematics and Statistics, Arizona State University, Tempe, AZ 85287, USA \\ ${ }^{4}$ Department of Aerospace Engineering, Texas A\&M University, College Station, TX 77843, USA
}

(Received 19 September 2007 and in revised form 23 January 2008)

The effects of harmonically oscillating the inner cylinder about a zero mean rotation in a Taylor-Couette flow are investigated experimentally and numerically. The resulting time-modulated circular Couette flow possesses a $Z_{2}$ spatio-temporal symmetry which gives rise to two distinct modulated Taylor vortex flows. These flows are initiated at synchronous bifurcations, have the same spatial symmetries, but are characterized by different spatio-temporal symmetries and axial wavenumber. Mode competition between these two states has been investigated in the region where they bifurcate simultaneously. In the idealized numerical model, the two flows have been found to coexist and be stable in a narrow region of parameter space. However, in the physical experiment, neither state has been observed in the coexistence region. Instead, we observe noise-sustained flows with irregular time-dependent axial wavenumber. Movies are available with the online version of the paper.

\section{Introduction}

Taylor-Couette flow, consisting of a viscous fluid confined in the gap between two rotating cylinders, has been a cornerstone in the development of hydrodynamic instability theory since the ground-breaking experimental and theoretical work of Taylor (1923). For low angular velocities, the flow is steady and purely azimuthal. This basic state is known as circular Couette flow. When the outer cylinder (of radius $r_{o}$ ) is at rest and the angular velocity of the inner cylinder (of radius $r_{i}$ ) exceeds a critical value, circular Couette flow becomes unstable and axisymmetric cells develop, separated by radial jets of angular momentum emanating from the cylinder boundary layers. This flow pattern, known as Taylor vortex flow, is steady and periodic in the axial direction.

Time-harmonic modulations of the inner cylinder rotation have been and continue to be of much interest. The original motivation for studying the effects of modulations was the experimentally observed threshold shift to higher mean rotation for the onset of sustained Taylor vortices (Donnelly 1964). Hall (1975), neglecting curvature effects by taking the small-gap limit $\eta=r_{i} / r_{o} \rightarrow 1$, performed a perturbation analysis for small modulation amplitudes and frequencies, showing that in this limit

$\dagger$ Present address:Department of Aerospace Engineering, Texas A\&M University, College Station TX 77843, USA. 
the modulations slightly destabilize the basic state. His results were confirmed by the Floquet analysis (also in the narrow-gap limit) of Riley \& Laurence (1976), contradicting the experimental results of Donnelly (1964). In an attempt to shed light on the nature of this discrepancy, Carmi \& Tustaniwskyj (1981) extended the Floquet analysis to finite values of the radius ratio, i.e. including curvature effects. However, their results showed a much larger degree of destabilization than the previous theoretical and computational studies of Hall (1975) and Riley \& Laurence (1976), contradicting all of the previous experimental and theoretical work on the problem. Barenghi \& Jones (1989) performed nonlinear computations of the NavierStokes equations for finite gap and confirmed the results of Hall (1975) and Riley \& Laurence (1976). They noted that the computations of Carmi \& Tustaniwskyj (1981) were performed with too large a time-step, so that in the low-frequency limit they were unable to properly reproduce the exponential growth and decay of the perturbations during a modulation period. Following Hall (1983), who showed that in the lowfrequency limit the most relevant perturbations are not periodic solutions of the equations of motion, Barenghi \& Jones (1989) introduced a low level of noise into their computations and obtained qualitative agreement with Ahlers' experimental results which were published as an appendix to Barenghi \& Jones (1989). They concluded that the discrepancies regarding stability limits were due to noise-induced difficulties in experimentally determining the onset of instability.

The Floquet analysis of Riley \& Laurence (1976) in the narrow-gap limit showed that for zero-mean modulations of the inner cylinder, two different time-periodic Taylor vortex flows compete. For low frequencies the flow is characterized by a reversal of the sign of the radial and axial velocities every half-period, whereas for higher frequencies the radial and axial velocities pulse twice during a cycle without changing sign. Nevertheless, none of the subsequent Floquet analyses for finite values of the radius ratio (Carmi \& Tustaniwskyj 1981; Barenghi \& Jones 1989) detected competition between the two different modes. This was attributed to a failure of the narrow-gap limit in the modulated Taylor-Couette system (Carmi \& Tustaniwskyj 1981). More recently, Youd, Willis \& Barenghi (2003) performed nonlinear computations for a medium gap $(\eta=0.75)$ and confirmed the existence of these modes, terming them reversing and non-reversing Taylor vortex flow, respectively. Consequently, it was not clear whether the linear stability results of Riley \& Laurence (1976) extended to the case of finite radius ratio $\eta$ or if one or both modes were due to nonlinear growth of finite-amplitude perturbations.

In this paper, we have performed a Floquet analysis of the basic state and found that both reversing and non-reversing flows are due to linear instabilities of the basic state, confirming the results of Riley \& Laurence (1976). In fact, the instabilities are pitchfork-of-revolution bifurcations in which the axial translation invariance is broken. For non-reversing flow this is the only symmetry which is broken, whereas the pitchfork-of-revolution bifurcation leading to reversing flow also breaks the half-period-flip spatio-temporal symmetry under which the basic state is invariant. Hydrodynamic systems with the same symmetries that bifurcate to two different competing periodic modes have also been found numerically and experimentally in periodically driven cavity flow (Marques, Lopez \& Blackburn 2004; Vogel, Hirsa \& Lopez 2003) and in the wake of a circular cylinder (Barkley \& Henderson 1996; Williamson 1996). In those problems, the bifurcating states were named modes A and $\mathrm{B}$, and we shall adopt this nomenclature in the present problem, rather than using the terms reversing and non-reversing. Here, we not only characterize the spatiotemporal properties of the bifurcated flows, but also investigate mode competition in 


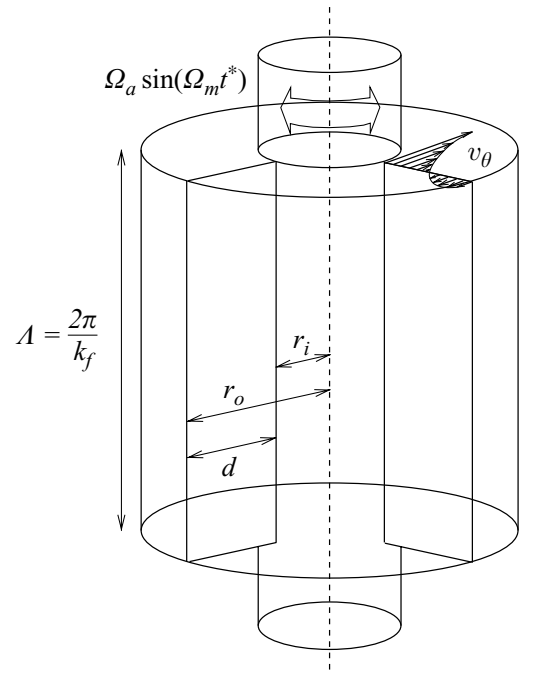

FIgURE 1. Schematic of modulated Taylor-Couette flow.

a neighbourhood of the codimension-two point where they bifurcate simultaneously, using both nonlinear simulations and laboratory experiments.

\section{Numerical formulation and symmetries}

Consider an incompressible fluid of kinematic viscosity $v$ contained between two concentric cylinders of inner and outer radii $r_{i}$ and $r_{o}$. The inner cylinder rotates at an angular velocity $\Omega\left(t^{*}\right)$ which is modulated harmonically in time about a zero mean

$$
\Omega\left(t^{*}\right)=\Omega_{a} \sin \left(\Omega_{m} t^{*}\right) .
$$

Figure 1 is a schematic of the system geometry. The dimensionless dynamical parameters are the modulation amplitude, given by the Reynolds number $R e_{a}=$ $\Omega_{a} d r_{i} / \nu$, and the modulation frequency $\omega=\Omega_{m} d^{2} / \nu$, where $d=r_{o}-r_{i}$ is the gap between cylinders. The geometry of the annulus is defined by the radius ratio $\eta=r_{i} / r_{o}$, which we fix at $\eta=0.5$ in the computations to match that of the experimental apparatus. For the Floquet analysis, the cylinders are assumed to be infinite, whereas the nonlinear computations are performed in a long periodic annulus with axial period 41.89 times the annular gap.

Using $d$ and $d^{2} / v$ as the space and time scales, the non-dimensional Navier-Stokes equations are

$$
\partial_{t} \boldsymbol{v}+(\boldsymbol{v} \cdot \nabla) \boldsymbol{v}=-\nabla p+\Delta \boldsymbol{v}, \quad \nabla \cdot \boldsymbol{v}=0 .
$$

The boundary conditions in cylindrical coordinates are

$$
\boldsymbol{v}\left(r_{i}, \theta, z, t\right)=(0, \operatorname{Re}(t), 0), \quad \boldsymbol{v}\left(r_{o}, \theta, z, t\right)=\mathbf{0},
$$

where $\operatorname{Re}(t)=R e_{a} \sin (\omega t)$ is the instantaneous Reynolds number. The resulting modulated Couette flow $(\mathrm{mC})$ is purely azimuthal and synchronous with the imposed harmonic oscillations

$$
\boldsymbol{v}_{m C}=\left(0, \operatorname{Re}_{a} f(r) \sin (\omega t+\alpha(r)), 0\right),
$$


where $f(r) \mathrm{e}^{\mathrm{i} \alpha(r)}$ is the solution of a second-order ODE satisfying the boundary conditions (Lopez \& Marques 2002); $f$ can be expressed in terms of modified Bessel functions, but here it has been computed using collocation at Gauss-Lobatto points.

The linear stability of $\mathrm{mC}$ (2.4) has been determined by Floquet analysis following Lopez \& Marques (2002). In order to investigate the dynamics of the bifurcated flows beyond onset, nonlinear computations of the Navier-Stokes equations are required. The velocity field is written as

$$
\boldsymbol{v}(r, \theta, z, t)=\boldsymbol{v}_{m C}(r, t)+\boldsymbol{u}(r, \theta, z, t),
$$

and the perturbation $\boldsymbol{u}$ is discretized with a solenoidal spectral approximation

$$
\boldsymbol{u}_{\mathrm{s}}(r, \theta, z, t)=\sum_{l=-L}^{L} \sum_{n=-N}^{N} \sum_{m=0}^{M} a_{l n m}(t) \mathrm{e}^{\mathrm{i}\left(l k_{f} z+n \theta\right)} \boldsymbol{u}_{l n m}(r),
$$

which satisfies axially periodic boundary conditions

$$
\boldsymbol{v}(r, \theta, z, t)=\boldsymbol{v}(r, \theta, z+\Lambda, t),
$$

with $\Lambda=2 \pi / k_{f}$.

The spectral scheme is obtained by substituting (2.5) in (2.2) and projecting over a suitable set of test solenoidal fields. This projection yields a system of ODEs for $a_{l n m}(t)$ which is integrated in time with a semi-implicit method, using backward differences for the diffusion term and polynomial extrapolation for the advective term (see Avila, Meseguer \& Marques 2006, for further details on the nonlinear numerical scheme).

It is very useful, in the time evolution, to monitor the kinetic energy associated with each axial Fourier mode in the spectral approximation:

$$
E_{l}(t)=\frac{1}{2 V} \int_{0}^{\Lambda} \mathrm{d} z \int_{0}^{2 \pi} \mathrm{d} \theta \int_{r_{i}}^{r_{o}} \boldsymbol{u}_{l}^{*} \cdot \boldsymbol{u}_{l} r \mathrm{~d} r
$$

where $V$ is the volume of the annular domain, and $\boldsymbol{u}_{l}$ is the $l$ th axial component of the perturbation field $\boldsymbol{u}$

$$
\boldsymbol{u}_{l}=\mathrm{e}^{\mathrm{i} l k_{f} z} \sum_{n=-N}^{N} \sum_{m=0}^{M} a_{l n m}(t) \mathrm{e}^{\mathrm{i} n \theta} \boldsymbol{u}_{l n m}(r) .
$$

In order to account for several harmonics of the most unstable Fourier modes, truncations of up to $(L, M, N)=(300,20,10)$ in (2.6) were used in the present investigations. Nevertheless, for the parameter regimes investigated the flows were found to be axisymmetric, in agreement with the experiments. Hence, only the $n=0$ azimuthal mode makes a contribution to (2.9). The accuracy of the results was checked by increasing the resolution to $(L, M, N)=(420,24,10)$, and the difference between the two truncations is less than one part in a thousand in the computation of the bifurcation curves $\operatorname{Re}_{a}(\omega)$ shown in figure 14 .

\subsection{Wavenumber selection in axially periodic flows}

In a linear stability analysis, the axial wavenumber of the perturbation $\boldsymbol{u}$ varies continuously. However, in the nonlinear computations the axial direction is treated as being periodic and a suitable fundamental axial wavenumber $k_{f}$ in (2.6) has to be selected such that the discretization resolves those axial modes responsible for the instability. In order to illustrate how to choose $k_{f}$, let us consider the infinite cylinders case with the inner cylinder rotating at a constant angular speed, $R e_{0}$, and 
(a)

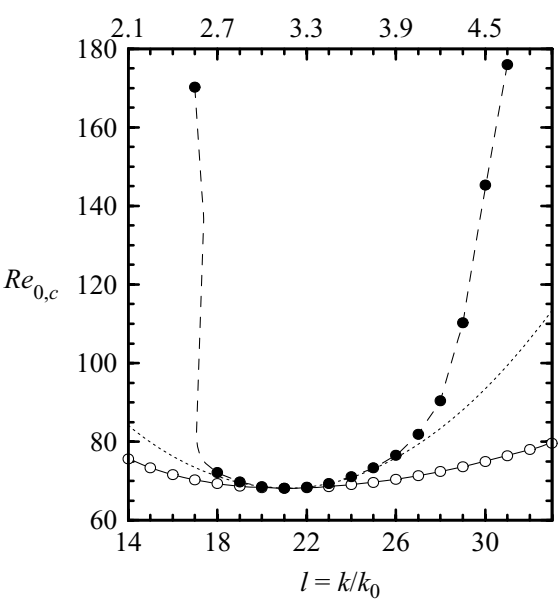

(b)

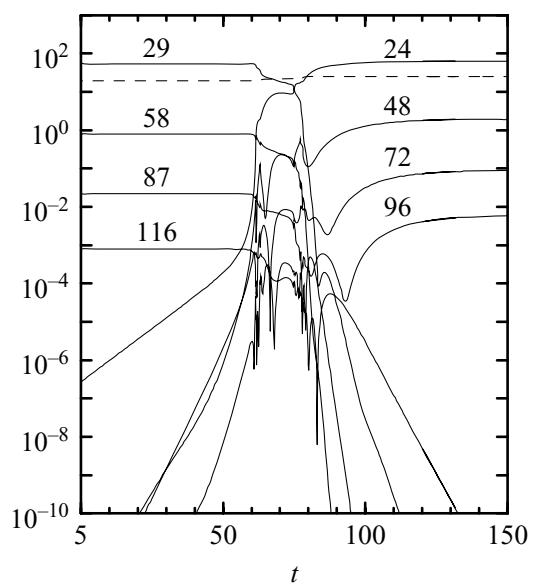

Figure 2. (a) Neutral (solid line) and Eckhaus (dashed line) stability curves for Couette flow with $\eta=0.5$ and infinite cylinders (computations by Riecke \& Paap 1986). The open and filled circles correspond to the points in these curves with axial wavenumbers in our spectral approximation (2.6), i.e. $k=l k_{f}$ with $k_{f}=0.15$. The dotted line corresponds to the third-order Ginzburg-Landau approximation of the Eckhaus curve. (b) Solid lines show the kinetic-energy time series of the $l=24,29$ axial Fourier modes, and their three first harmonics, in the transition from the Eckhaus unstable $\mathrm{TVF}_{58}$ to $\mathrm{TVF}_{48}$ at $R e_{0}=100$. The dashed line corresponds to the $l=0$ mode.

the outer cylinder stationary. For $\eta=0.5$, circular Couette flow becomes linearly unstable via a pitchfork-of-revolution bifurcation to axisymmetric Taylor vortex flow (TVF) at $R e_{0, c}=68.19$, with critical axial wavenumber $k_{0, c}=3.162$. For $R e_{0}>R e_{0, c}$ a continuous wave-vector band of TVF solutions bifurcates from circular Couette flow. The width of this band at a particular $R e_{0}$ is given by the neutral stability curve of figure 2(a) (solid line). However, apart from the solution with $k_{0, c}$ at $R e_{0}$, the other solutions are born unstable and stabilize for higher $R e_{0}$ upon crossing the so-called Eckhaus curve. Figure 2(a) shows the Eckhaus curve as obtained by Riecke $\&$ Paap (1986) assuming continuous axial wavenumber $k$ (dashed curve), and its classic Ginzburg-Landau estimate (dotted curve).

In the nonlinear computations the spectrum of axial wavenumbers is discrete owing to the imposed periodic boundary conditions (2.7). Here, we have used a small fundamental axial wavenumber $k_{f}=0.15$ which corresponds to a periodic annular domain of axial wavelength $\Lambda=2 \pi / k_{f}=41.89$. With this choice, the most unstable Fourier mode in our spectral expansion (2.6) is $l=21$, corresponding to an axial wavenumber $k=l k_{f}=3.15$, which is very close to the critical value $k_{c}$. Figure 3 shows contours of the Stokes streamfunction $\psi$ and azimuthal vorticity $\omega_{\theta}$ for nonlinear TVF at $R e_{0}=100$ with $k=3.15$. The wavelength of the resulting periodic pattern is $\lambda=2 \pi / k=1.995$, which corresponds to approximately square counter-rotating Taylor cells. These cells are separated by inflow and outflow jets of angular momentum, $r v_{\theta}$, emanating from the inner and outer cylinder, respectively. When considering the full axial domain, $z \in[0, \Lambda]$, the corresponding TVF in figure 3 spans $\Lambda /(\lambda / 2)=2 l=42$ cells, $\mathrm{TVF}_{42}$. For $R e_{0}>R e_{0, c}$ a discrete family of Taylor vortex flows with different $l$ bifurcate supercritically from circular Couette flow at the open circles in figure $2(a)$. These TVF are unstable at onset as circular Couette 


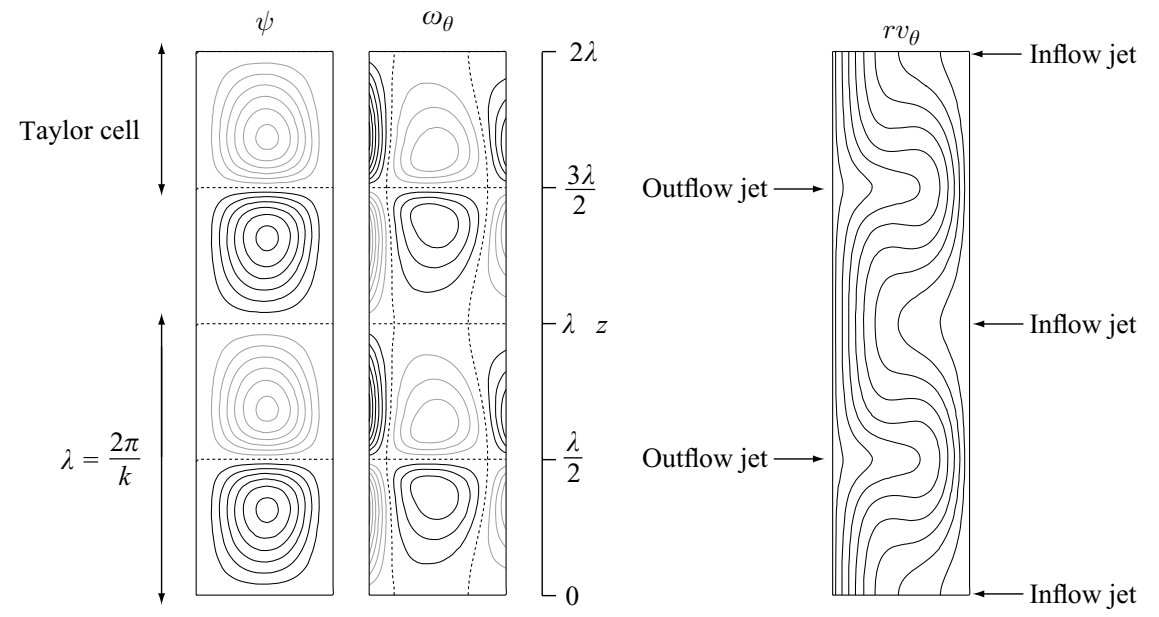

FIGURE 3. Taylor vortex flow $\left(\mathrm{TVF}_{42}\right)$ at $R e_{0}=100$ with axial wavenumber $k=3.15$. Contours of the streamfunction $\psi$ and azimuthal vorticity $\omega_{\theta}$ are shown in a meridional cross-section. Vortex lines ( $r v_{\theta}$ contours) show the outflow and inflow jets separating the Taylor cells. Black (grey) contours correspond to positive (negative) values, showing $r \in[1,2]$ and $z \in[0,2 \lambda]$.

flow is already unstable to $\mathrm{TVF}_{42}$, and they become stable at secondary bifurcations (Tuckerman \& Barkley 1990). These secondary bifurcations are indicated by the filled circles in figure 2(a) and coincide very well with the Eckhaus curves computed by Riecke \& Paap (1986) and the large-aspect-ratio experiments of DominguezLerma, Cannell \& Ahlers (1986). Here, the Eckhaus instability curve was determined using nonlinear computations. For example, starting at $R e_{0}=100$ with a random perturbation in the Fourier subspace spanned by mode $l=29$, with corresponding $k=4.35$, the perturbation is evolved in time, resulting in steady $\mathrm{TVF}_{58}$. Then, a small perturbation to the modes $l=21, \ldots, 28$ is introduced and their kinetic energy (2.8) is monitored in time. In this case, $\mathrm{TVF}_{58}$ is Eckhaus unstable and evolves to $\mathrm{TVF}_{48}$, i.e. with $l=24$ and $k=3.6$. This transition is illustrated in figure $2(b)$, showing the kinetic energy of the axial Fourier modes $l=24,29$ and their first three harmonics. The process is repeated with increasing $R e_{0}$ in order to detect the point of bifurcation. The use of a small fundamental axial wavenumber $k_{f}$ is essential for capturing the nonlinear competition between modes as the Eckhaus curve is crossed.

The implementation of small $k_{f} \ll k_{c}$ is very useful in modulated Taylor-Couette flow, where the bifurcating modes (reversing and non-reversing TVF) are characterized by distinct axial wavenumbers. In previous numerical studies with periodic boundary conditions (Youd, Willis \& Barenghi 2003, 2005), the evolution of the flows was computed by fixing $k_{f}=k_{c}$. Although this latter approach allows one to investigate the stability to non-axisymmetric perturbations (wavy vortex flows), it is unable to consider interactions between flows with different axial wavenumbers (except for the exceptional cases that they are multiples of $k_{c}$ ).

\subsection{Symmetries}

The governing equations and boundary conditions are invariant to the $K_{z}$ reflection $z \rightarrow-z$ and to translations $T_{a}$ along the $z$-axis. Owing to the imposed axial periodicity of wavelength $\Lambda, T_{a}$ generates an $S O(2)$ symmetry group. Note that as $K_{z}$ and $T_{a}$ do not commute (in fact $K_{z} T_{a}=T_{-a} K_{z}$ ), they together generate an $O(2)$ symmetry group which is not the direct product of $S O(2)$ and $Z_{2}$, but the semidirect product 
$O(2)=S O(2) \rtimes Z_{2}$. The elements of $Z_{2}$ are the identity and $K_{z}$. In the azimuthal direction the governing equations are invariant to arbitrary rotations $R_{\alpha}$ which generate another $S O(2)$ symmetry group. Since both $T_{a}$ and $K_{z}$ commute with $R_{\alpha}$, these operations generate the group $\mathscr{G}_{0}=S O(2) \times O(2)$ of the spatial symmetries of the system. The actions of these symmetries on the velocity field are

$$
\begin{aligned}
& T_{a} \boldsymbol{v}(r, \theta, z, t)=\boldsymbol{v}(r, \theta, z+a, t), \\
& K_{z} \boldsymbol{v}(r, \theta, z, t)=\left(v_{r}, v_{\theta},-v_{z}\right)(r, \theta,-z, t), \\
& R_{\alpha} \boldsymbol{v}(r, \theta, z, t)=\boldsymbol{v}(r, \theta+\alpha, z, t) .
\end{aligned}
$$

The basic state (2.4) is invariant under $\mathscr{G}_{0}$. Moreover, when modulations about a zero mean are considered, there is an additional spatio-temporal symmetry $S$, which consists of a time evolution of half a modulation period followed by the reflection $K_{\theta}$ on the meridional plane $\theta=0$ (acting as $\theta \rightarrow-\theta$ ), whose action on the velocity is

$$
S(\boldsymbol{v})(r, \theta, z, t)=K_{\theta}(\boldsymbol{v})(r, \theta, z, t+T / 2)=\left(v_{r},-v_{\theta}, v_{z}\right)(r,-\theta, z, t+T / 2),
$$

where $T=2 \pi / \omega$. If $S$ were purely spatial, i.e. the reflection $K_{\theta}$, then $S$ and $R_{\alpha}$ would generate the orthogonal group, $O(2)=S O(2) \rtimes Z_{2}$, where the elements of $Z_{2}$ would be $K_{\theta}$ and the identity. Since $S$ is a space-time symmetry, $S$ and $R_{\alpha}$ generate a space-time symmetry group isomorphic to $O(2)$, which we term $O(2)^{S T}=S O(2) \rtimes Z_{2}^{S T}$, where now the elements of $Z_{2}$ are $S$ and the identity. Therefore, the complete symmetry group of the system modulated about a zero mean is $\mathscr{G}=O(2)^{S T} \times O(2)$ and the basic state (2.4) is invariant under $\mathscr{G}$. When the dynamics preserve the axisymmetry, as is the case over large regions in parameter space, the rotations $R_{\alpha}$ do not play any dynamic role (they act trivially on the solutions of the governing equations), and in the axisymmetric subspace the symmetry group of the problem reduces to $\mathscr{G}=Z_{2}^{S T} \times O(2)$.

Generally, the stability of $T$-periodic flows is determined by Floquet analysis, considering their Poincaré map

$$
x_{0} \longmapsto \mathscr{P}\left(x_{0}\right)=\phi\left(t_{0}+T ; x_{0}, t_{0}\right),
$$

where $\phi\left(t_{0}+t ; x_{0}, t_{0}\right)$ is the solution of the governing equations at time $t$ with initial conditions $\left(x_{0}, t_{0}\right)$ in a neighbourhood of the periodic orbit. However, for systems with $Z_{2}$ spatio-temporal symmetry, the Poincare map is the square of the half-period-flip map

$$
x_{0} \longmapsto \mathscr{H}\left(x_{0}\right)=K_{\theta} \phi\left(t_{0}+T / 2 ; x_{0}, t_{0}\right) .
$$

In these cases, the eigenvalues of $\mathscr{P}$ are $\mu_{\mathscr{P}}=\mu_{\mathscr{H}}^{2}$, where $\mu_{\mathscr{H}}$ is the corresponding eigenvalue of $\mathscr{H}$. The action of $S$ has important implications for the dynamics of the bifurcated solutions which cannot be detected by studying the stability of $\mathscr{P}$ alone. Marques et al. (2004) derived normal forms for codimension-one and -two bifurcations in systems with $\mathscr{G}=Z_{2}^{S T} \times O(2)$, for both $\mathscr{H}$ and $\mathscr{P}$.

\section{Experimental apparatus and methods}

\subsection{Apparatus}

The apparatus, shown schematically in figure 4 , is a reassembly of the original apparatus used by Takeuchi \& Jankowski (1981) for the study of spiral Poiseuille flow. It has been thoroughly overhauled with a new drive system, piping system, and digital-image acquisition system. The through-flow feature, although not used in 


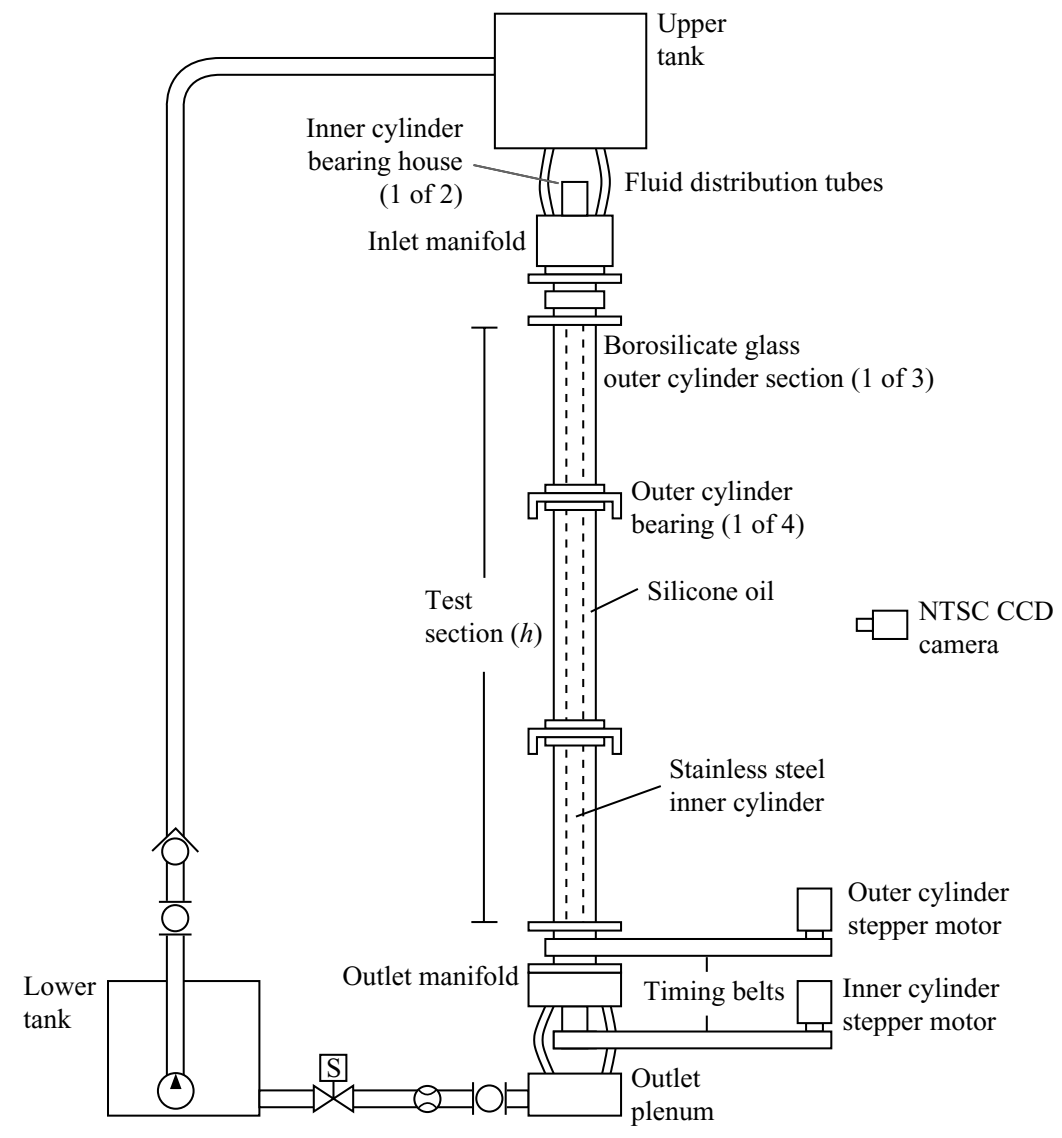

FIGURE 4. Schematic of the experimental apparatus. Overall height is approximately $4.5 \mathrm{~m}$.

the present experiments, affects the boundary conditions at the ends of the annulus. Unlike most other Taylor-Couette apparatus, the ends are not rigid. The ends of the annulus open into the inlet and outlet manifolds and so the boundary conditions at the ends are not clearly defined. Moreover, the inlet and outlet are not exactly symmetrical and so the reflection symmetry about the annulus half-height is imperfect.

The inner cylinder is a stainless-steel pump shaft having a radius of $r_{i}=25.40 \pm$ $0.01 \mathrm{~mm}$. Three precision borosilicate glass tube sections form the outer cylinder, each with a constant inner radius of $r_{o}=50.80 \pm 0.05 \mathrm{~mm}$ and nominal length $700 \mathrm{~mm}$. After alignment, the maximum run-out of the inner cylinder is $0.03 \mathrm{~mm}$ at the midsection. The radius ratio is thus $\eta=r_{i} / r_{o}=0.500 \pm 0.003$ over one glass section. The length of the outer cylinder is $h=2.93 \mathrm{~m}$, which corresponds to an aspect ratio of $\Gamma=h / d=115 \pm 1$. There are four bearings along the length of the test section, each having six degrees of freedom for alignment. Including local deviations near the bearings, the variation in gap width over the entire length is less than $3.3 \%$. The entire test section is environmentally sealed to reduce the effect of convection from installation considerations such as the room heat pump.

The inner and outer cylinders are rotated independently by Industrial Devices Corporation (IDC) P21V stepper motors. Each stepper motor connects to a Bayside PX60-10 10:1 gearbox which connects via a timing belt to the inner and outer cylinders. The total gear ratios of the inner and outer cylinders to 
(a)

(b)

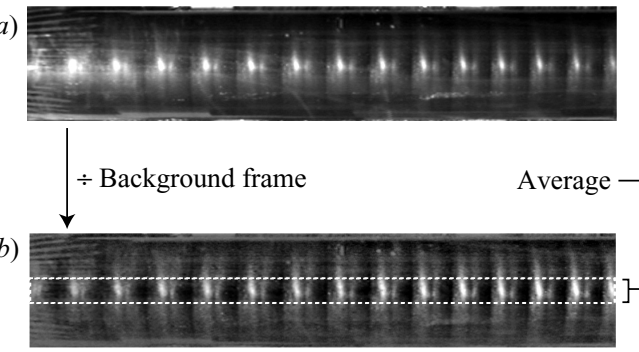

Relative

(c) intensity

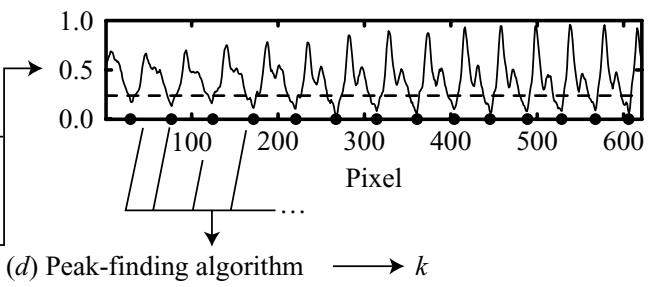

FiguRE 5. Data post-processing flow chart.

each respective motor shaft are 200:7 and 240:7, respectively. The motors are controlled by IDC SmartStep23 integrated controllers. MCG E9 optical rotary encoders (8000 tics/revolution, post-quadrature) are connected to the motor shaft and measure position. The inner cylinder is rotated using a discretized sine wave with 66 increments per cycle. The deviation from a pure sine wave is less than $0.2 \%$.

The experimental fluid is $10 \mathrm{~mm}^{2} \mathrm{~s}^{-1}$ Dow Corning 200 Polydimethylsiloxane. Type $\mathrm{T}$ thermocouples are used to monitor the temperature in the upper and lower reservoirs and resistance temperature detector probes measure the temperature inside and outside the enclosure. The thermocouples connect to a National Instruments PCI-6035E data-acquisition (DAQ) card through a National Instruments SC-2345 signal conditioning unit, while the temperature probes connect to a Hanyoung NX-9 process controller with 4-20 mA output that is in turn connected to the DAQ card.

The difference in temperature between the two reservoirs is nominally less than $0.5 \mathrm{~K}$. The variation inside the test-section enclosure is sinusoidal about a mean within $0.1 \mathrm{~K}$ and tracks the cycling room temperature which varies within $0.4 \mathrm{~K}$. The fluid is cycled between runs and the temperatures are allowed to settle before starting an experiment. As such, the mean temperature of the chamber is considered to be the experimental temperature for viscosity determination. The temperature dependence of the viscosity was measured to within $0.3 \%$ using an Ubbelohde viscometer manufactured by Cannon Instrument Company.

Including all of the effects above, the total uncertainties in $R e_{a}$ and $\omega$ are $1.4 \%$ and $2.2 \%$, respectively.

\subsection{Methods}

Space-time images were obtained by observing an axial cross-section of the flow using a Sony XC-ST50 Monochrome CCD video camera connected to a Linux Media Labs LMLBT44 capture card. The size of a pixel is typically $0.04 d$ with no measurable lens distortion over the 640-pixel-wide frame. The technique, illustrated in figure 5, follows that of Linek \& Ahlers (1998) and numerous others. Reflective flakes (Flamenco Superpearl $120 \mathrm{C}+$, mica coated with $\mathrm{TiO}_{2}$ ) were illuminated by tungsten lighting to visualize the vortex structure and measure the wavenumber. Detailed investigations of how such flakes behave in a flow were performed by Savaş (1985) and Gauthier, Gondret \& Rabaud (1998).

A camera was oriented to observe the flow in the middle section (about $24 d$ in axial extent). At the start of an experiment, a series of background images were taken with the inner cylinder at rest. The apparatus was then operated at fixed $R e_{a}$ and $\omega$ while acquiring image data at a rate of between 1 and 3 images per second. Data at 
different parameter values were either acquired by a step change in the parameters, or from rest.

The intensity distribution in each frame (figure $5 a$ ) is divided, pixel by pixel, by the corresponding intensity distribution of the background image. A 12-pixel-wide slice is taken from the centre of the resulting image (figure $5 b$ ) and averaged over its width. These averaged slices are assembled into a time history and normalized. The uncertainty in the normalized intensity due to sensor noise is approximately $1 \%$.

In order to determine the axial wavenumber at a given time, the pixel intensities of a particular slice are low-pass filtered and a peak-finding algorithm determines the local minima below a threshold shown as a dashed line in figure 5(c). The minima correspond to axial locations (at the outer cylinder) of the radial inflow jets separating the Taylor cells. In the parameter regime investigated, the uncertainty in the (nondimensionalized) wavenumber is 0.1 . Further technical details of the experimental technique are presented in Belisle (2007).

\section{Types A (reversing) and B (non-reversing) Taylor vortex flows}

\subsection{Floquet analysis of the modulated Couette flow}

Codimension-one bifurcations are generically obtained when a single parameter is varied. For modulated Couette flow $(\mathrm{mC})$, the possible codimension-one bifurcations correspond to Floquet multipliers of the Poincare map $\mu_{\mathscr{P}}=1$ or the complexconjugate pair $\mu_{\mathscr{P}}=\exp ( \pm \mathrm{i} \theta)$. The case of $\mu_{\mathscr{P}}=-1$ is the period-doubling bifurcation which is inhibited by the space-time $Z_{2}$ symmetry $S$ (Swift \& Wiesenfeld 1984). Furthermore, the synchronous case $\mu_{\mathscr{P}}=1$ comes in two flavours, one which breaks the symmetry $S$ and another which preserves it. These two cases are readily distinguished by using the half-period-flip map $\mathscr{H}$ for the Floquet analysis, where $\mu_{\mathscr{P}}=\mu_{\mathscr{H}}^{2}$, and $\mu_{\mathscr{H}}=+1$ is the $S$-preserving synchronous bifurcation and $\mu_{\mathscr{C}}=-1$ is the $S$-breaking synchronous bifurcation. The quasi-periodic case $\mu_{\mathscr{P}}=\exp ( \pm \mathrm{i} \theta)$, while also being generic, has not been observed for modulated Taylor-Couette flow in the parameter regimes studied so far. However, when the outer cylinder is modulated, Lopez \& Marques (2002) found some parameter regimes where the quasi-periodic case leads to modulated spiral flows.

Figure 6 shows the results of the Floquet analysis of the basic state $\mathrm{mC}$, using the half-period-flip map $\mathscr{H}$. The bifurcation curves $R e_{a}=R e_{a, c}(\omega)$ are shown in part $(a)$ of the figure. The solid curve is a pitchfork-of-revolution bifurcation corresponding to $\mu_{\mathscr{H}}=+1$, where mode $\mathrm{B}$ bifurcates from $\mathrm{mC}$, and the dashed curve is also a pitchfork-of-revolution bifurcation corresponding to $\mu_{\mathscr{H}}=-1$, where mode A bifurcates. These bifurcations break the continuous translational symmetry $T_{a}$, which is replaced by a discrete translational symmetry $T_{2 \pi / k_{c}}$, where $k_{c}(\omega)$ is the critical wavenumber at the bifurcation (see figure $6 b$ ). The two modes remain $T$-periodic but mode A breaks the $S$-symmetry and mode B preserves it.

The four regions in figure 6(a) are labelled with the states that exist in them (note that $\mathrm{mC}$ exists everywhere but is only stable in the region where it is labelled). Below both the $\mathrm{A}$ and $\mathrm{B}$ curves only the basic state $\mathrm{mC}$ exists and is stable. In the region above both the $\mathrm{A}$ and $\mathrm{B}$ curves, modes $\mathrm{A}$ and $\mathrm{B}$ coexist. At low modulation frequencies, mode $\mathrm{A}$ is the primary bifurcation (i.e. for a given $\omega$, $\mathrm{A}$ bifurcates from $\mathrm{mC}$ first as $R e_{a}$ is increased), and at large frequencies, mode $\mathrm{B}$ is primary. The point $\left(\omega, R_{a}\right)=(3.936,119.4)$ is a codimension-two point where the two bifurcations occur simultaneously. Figure 7 shows neutral stability curves for modulation frequencies $\omega=2.5,3.936$ and 6 . At $\omega=2.5$, the bifurcation to A is primary, occurring for a 
(a)

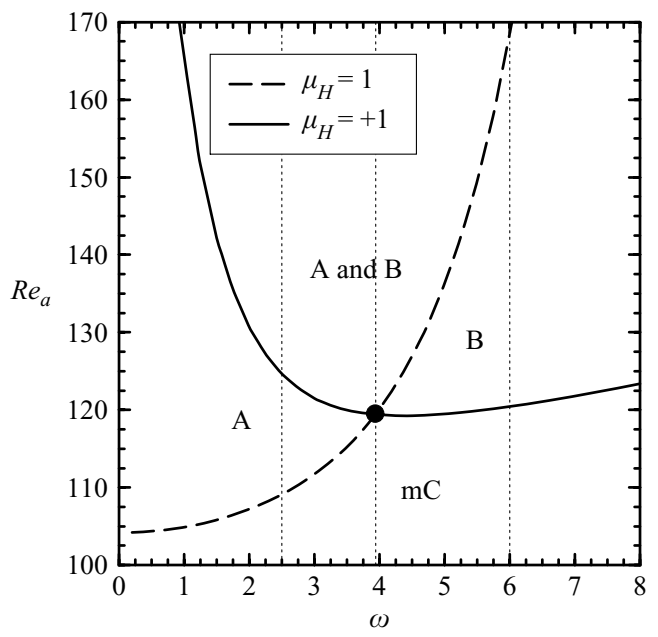

(b)

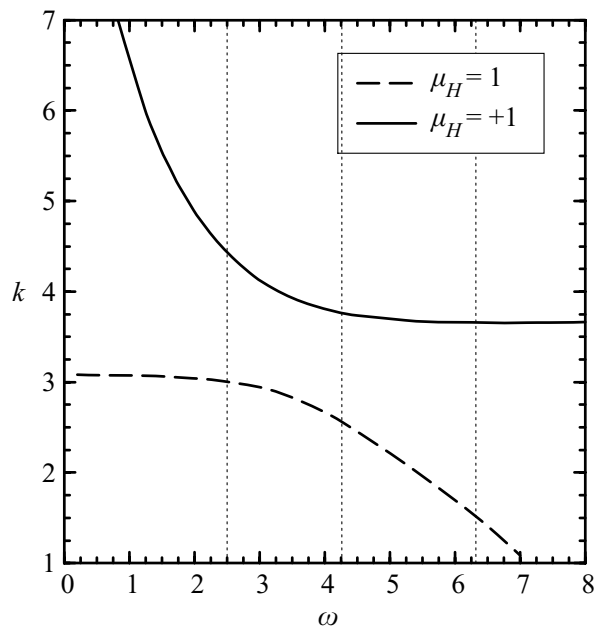

FIGURE 6. (a) Regime diagram obtained via Floquet analysis of the modulated Couette flow (2.4) using the half-period-flip map $\mathscr{H}$. The filled circle denotes the bicritical point $\left(\omega, R e_{a, c}\right)=(3.936,119.4)$. (b) Corresponding axial wavenumber $k_{c}(\omega)$ at criticality. The vertical dotted lines are the frequencies $\omega$ at which the marginal stability curves are shown in figure 7.
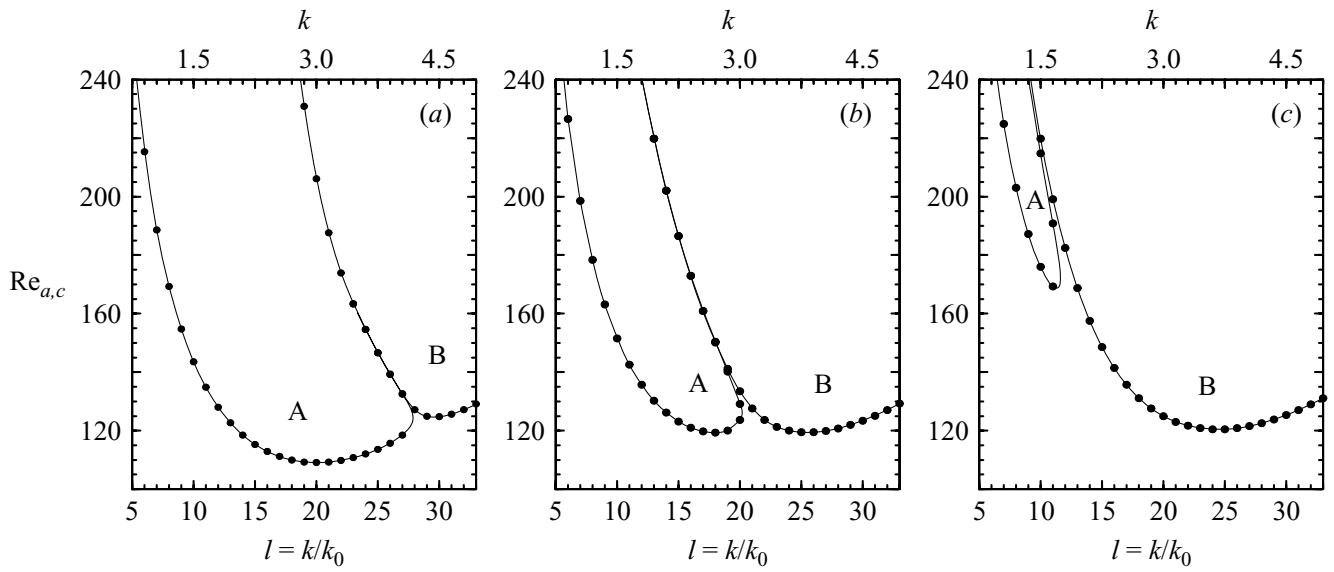

FiguRE 7. Neutral stability curves as computed with Floquet analysis for $(a) \omega=2.5$, $(b)$ $\omega=3.936$ and $(c) \omega=6$. The filled circles correspond to the discrete set of wavenumbers $k$ considered in the nonlinear computations such that $k \in[0.75,4.95]$.

wide range of wavenumbers. At $\omega=3.936$, both neutral stability curves have minima at $R e_{a, c}=119.4$, giving rise to the codimension-two bifurcation point. At $\omega=6$ the bifurcation to $\mathrm{A}$ is confined to a small range of wavenumbers and the bifurcation to $\mathrm{B}$ is primary. These linear stability results have the same features as the nonlinear computations of Youd et al. (2003) for $\eta=0.75$. Note that the neutral stability curves for $\mathrm{A}$ and $\mathrm{B}$ do not cross, so that at given $\left(\omega, R e_{a}\right)$ the wavenumber of the flow suffices to determine which state is realized in the nonlinear computations and experiments. However, comparing figures $7(a)$ and $7(c)$, it is clear that by changing either $R e_{a}$ or $\omega$ it is possible to obtain $\mathrm{A}$ and $\mathrm{B}$ with the same wavenumber. 

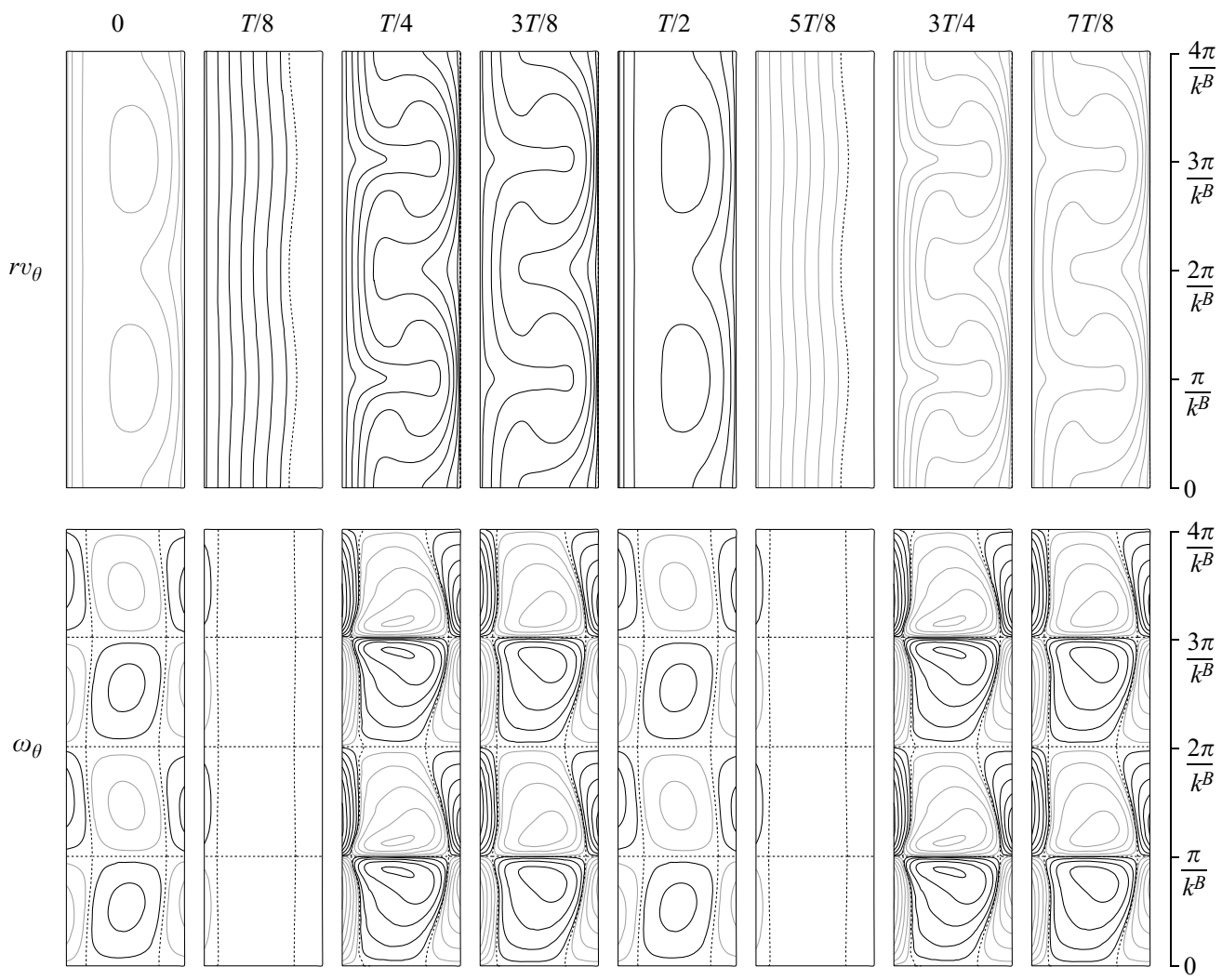

Figure 8. Type B (non-reversing) Taylor vortex flow at $\omega=6.1$ and $R e_{a}=240$ with wavenumber $k^{B}=3.4$. Quadratically spaced contours of angular momentum (top row) and azimuthal vorticity (bottom row) are shown in a meridional cross-section over a period $T=2 \pi / \omega$. Black (grey) contours correspond to positive (negative) values, showing $r \in[1,2]$ and $z \in\left[0,4 \pi / k^{B}\right]$. The snapshots have been taken after transients have vanished. In particular, a time shift of several periods $t \rightarrow t-N T$ has been applied. (Temporal behaviour is shown in Movie 1 available with the online version of the paper).

\subsection{Numerical characterization of the flows}

The Taylor-Couette system with modulations about a zero mean is characterized by two distinct phases, depending on the instantaneous value of $\operatorname{Re}(t)=R e_{a} \sin \omega t$. When $\operatorname{Re}(t)<\operatorname{Re}_{0, c}$ (the critical Reynolds number of steady Couette flow), the motion of the cylinder is subcritical and therefore all the perturbations decay. For $R e(t)>R e_{0, c}$ perturbations are amplified and vortices (Taylor cells) develop. Figure 8 shows contours of angular momentum $r v_{\theta}$ (top row) and azimuthal vorticity $\omega_{\theta}$ (bottom row) over a modulation period for state B at $\omega=6.1$ and $R e_{a}=240$ with wavenumber $k^{B}=3.4$. At $t=0$, which corresponds to $\operatorname{Re}(t)=0$, weak pairs of Taylor cells remain from the previous modulation cycle. At $t=T / 8$ the distribution of angular momentum is almost uniform as the Taylor cells from the previous cycle have almost completely decayed, but they soon re-develop and reach maximum amplitude at about $t=T / 4$ with a strong outflow jet of angular momentum erupting from the inner-cylinder boundary layer at $z=\pi / k^{B}$, and a weaker inflow jet from the outer-cylinder boundary layer at $z=0$. Subsequently, the cells decay until the rotation of the inner cylinder becomes supercritical in the opposite direction and the Taylor 
(a)

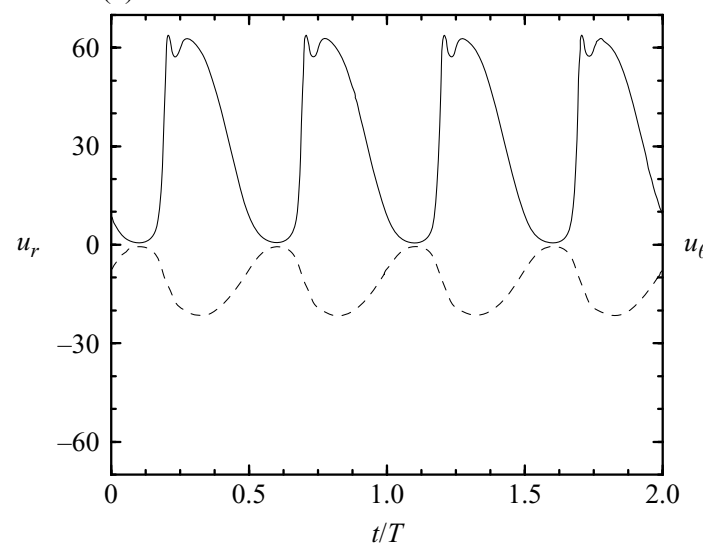

(b)

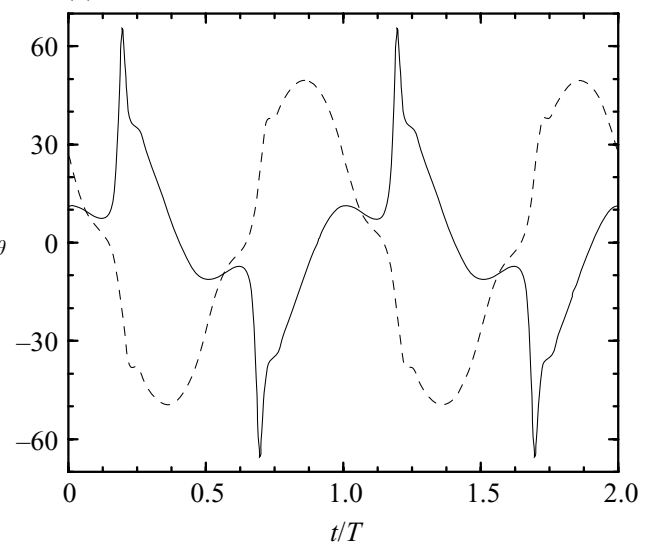

FIGURE 9. Evolution over two periods of $(a)$ the radial velocity and $(b)$ the azimuthal velocity components of the perturbation field (2.5) recorded at $(r, \theta, z)=\left(\left(r_{i}+r_{o}\right) / 2,0, \pi / k^{B}\right)$ (solid lines) and $(r, \theta, z)=\left(\left(r_{i}+r_{o}\right) / 2,0,0\right)$ (dashed lines) for state B in figure 8 . Note that the time axes have been normalized by the period $T$.

cells reappear at the same axial locations at $t=3 T / 4$. This indicates that a time evolution of $T / 2$ leaves the azimuthal vorticity invariant, with the axial locations of the inflow and outflow jets remaining unchanged. The time evolution of a half-period corresponds to a change of sign in the angular momentum, as it does for the basic state. It is therefore evident that the bifurcation to B preserves the spatio-temporal symmetry $S$. This can also be seen in figure 9 , showing the time series of the radial and azimuthal perturbation velocities, $u_{r}$ and $u_{\theta}$, at $(r, \theta, z)=\left(1.5,0, \pi / k^{B}\right)$ (solid lines) and $(r, \theta, z)=(1.5,0,0)$ (dashed lines). The radial velocity time series are $T / 2$ periodic, so that there is no reversal in the sense of circulation in the Taylor cells. However, the azimuthal velocity is $T$-periodic, with symmetrically opposed maxima and minima at $t$ and $t+T / 2$, i.e. $u_{\theta}(r, \theta, z, t)=-u_{\theta}(r, \theta, z, t+T / 2)$, and $S$ is preserved.

The time evolution of state A over a modulation period is illustrated in figure 10 , showing contours of angular momentum and azimuthal vorticity. The behaviour is similar to that of $\mathrm{B}$, although in this case it is clear that a time evolution of $T / 2$ does not leave the azimuthal vorticity invariant; the locations of the inflow and outflow jets are shifted by half an axial wavelength $\pi / k^{A}$ each half-period (compare snapshots at $t=T / 4$ and $t=3 T / 4$, for example). Note that this does not correspond to a reflection but to an axial shift of the pattern

$$
T_{\pi / k^{A}} \boldsymbol{v}(r, \theta, z, t)=\boldsymbol{v}\left(r, \theta, z+\pi / k^{A}, t\right) .
$$

The change in sign of angular momentum together with this half-wavelength axial shift indicates that the spatio-temporal symmetry $S$ has been broken. Nevertheless, A is invariant under a new $Z_{2}$ spatio-temporal symmetry $S^{\prime}$, consisting of $S$ composed with the axial translation $T_{\pi / k^{A}}$. Its action on the velocity field is

$$
S^{\prime}(\boldsymbol{v})(r, \theta, z, t)=\left(v_{r},-v_{\theta}, v_{z}\right)\left(r,-\theta, z+\pi / k^{A}, t+T / 2\right) .
$$

The replacement of $S$ by $S^{\prime}$ in the bifurcation to A is manifested in the time series of the radial and azimuthal perturbation components shown in figure 11. A time evolution of half a period changes the sign of the radial velocity, illustrating the exchange between outflow and inflow boundaries as a result of the axial shift. If this time evolution is 


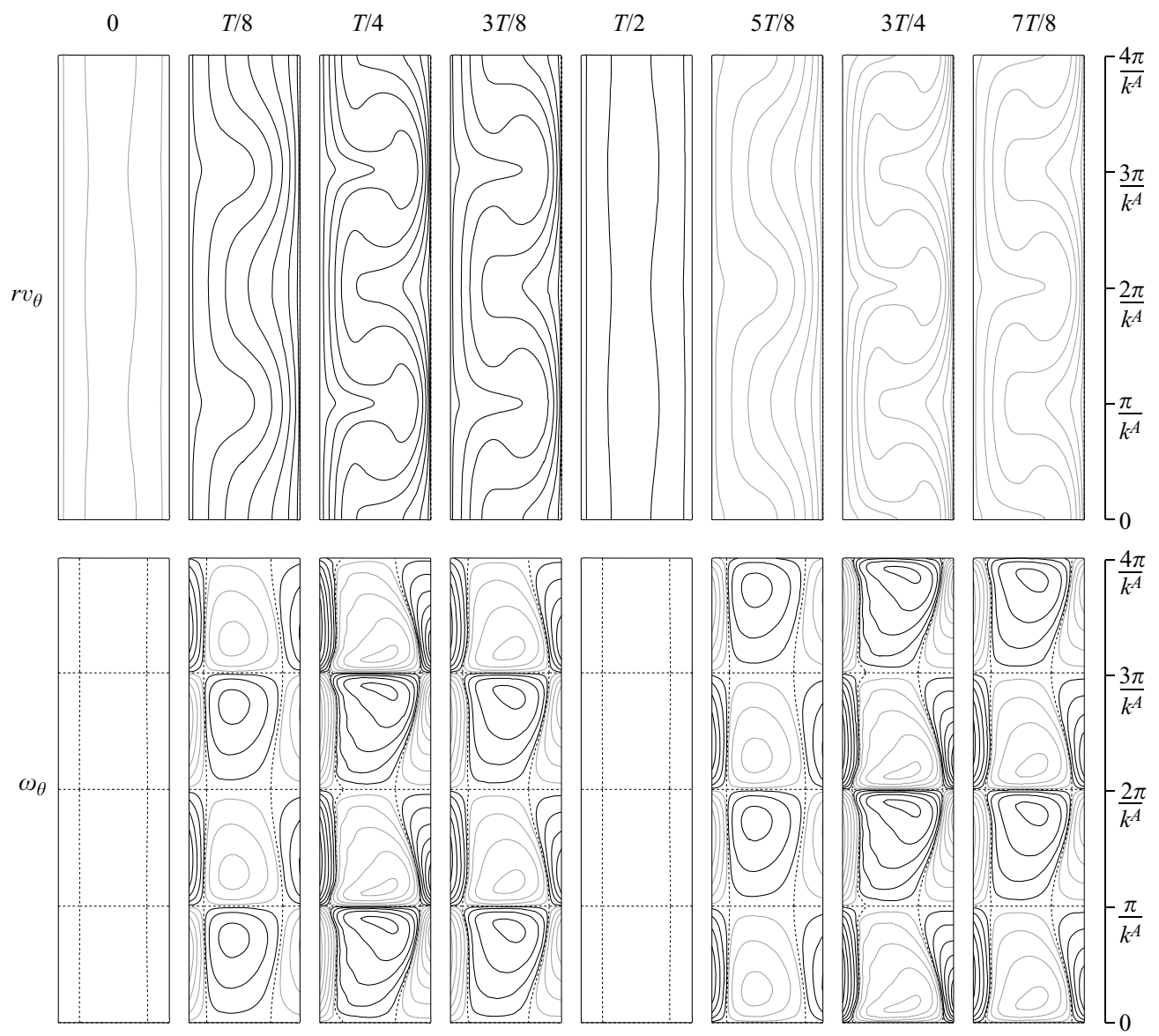

FIGURE 10. Type A (reversing) Taylor vortex flow at $\omega=1$ and $R e_{a}=240$ with wavenumber $k^{A}=3.0$. Quadratically spaced contours of angular momentum (top row) and azimuthal vorticity (bottom row) are shown in a meridional cross-section over a period $T$. Black (grey) contours correspond to positive (negative) values, showing $r \in[1,2]$ and $z \in\left[0,4 \pi / k^{A}\right]$. (Temporal behaviour is shown in Movie 2 available with the online version of the paper).

composed with $T_{\pi / k^{A}}$, which corresponds to swapping from solid to dashed line or vice-versa, then the radial velocity is unchanged. The azimuthal component is only invariant after further applying the reflection $K_{\theta}$. These symmetry considerations fully agree with the results from equivariant normal-form theory described in the Appendix.

\subsection{Experimental characterization of the flows}

The critical $R e_{a, c}$ from the Floquet analysis corresponds to the $R e_{a}$ at which the net growth of the most dangerous perturbation over a period is zero. For $R e_{a} \gtrsim R e_{a, c}$, the fraction of the period during which the motion of the cylinder is subcritical $\left(\operatorname{Re}(t)=R e_{a} \sin \omega t<R e_{0, c}\right)$ is large, and the vortices decay to energy levels which are limited by the level of background noise (Hall 1983; Barenghi \& Jones 1989). For larger $R e_{a}$, the signal-to-noise ratio is increased, and so the experimental results reported in this paper have been performed at $R e_{a}=240$, well beyond onset at $R e_{a, c} \approx 110$. At $R e_{a}=240$, the motion of the cylinder is subcritical for only $18.3 \%$ of the period, giving an acceptable signal-to-noise ratio which results in clean 
(a)

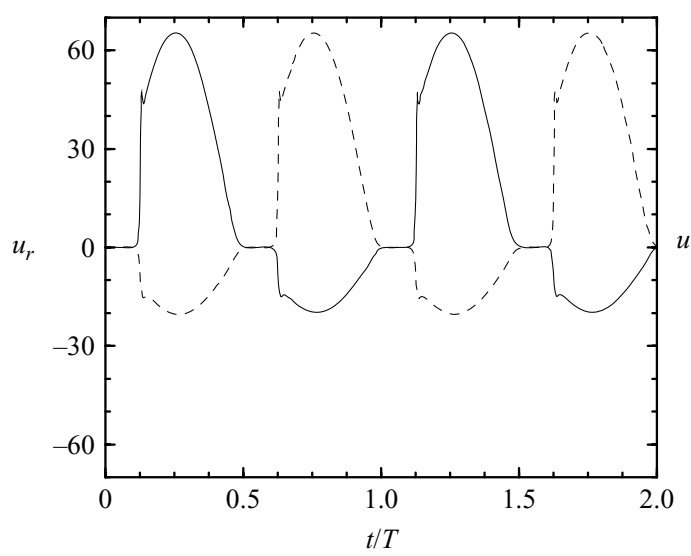

(b)

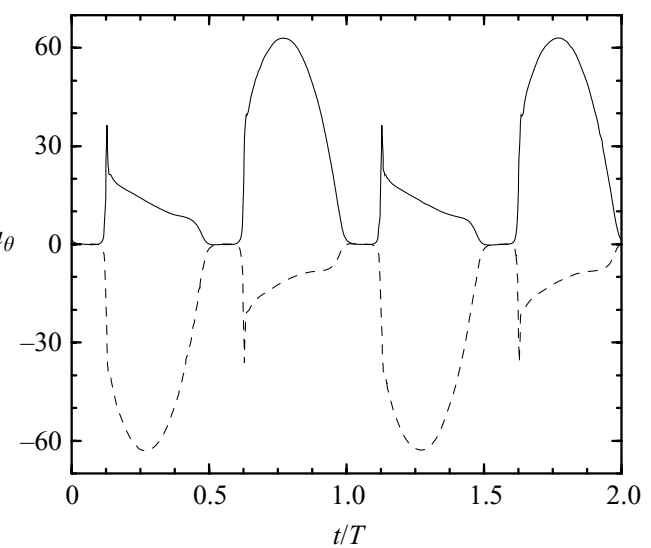

FiguRE 11. Evolution over two periods of $(a)$ the radial velocity and $(b)$ the azimuthal velocity components of the perturbation field $(2.5)$ recorded at $(r, \theta, z)=\left(\left(r_{i}+r_{o}\right) / 2,0, \pi / k^{A}\right)$ (solid lines) and $(r, \theta, z)=\left(\left(r_{i}+r_{o}\right) / 2,0,0\right)$ (dashed lines) for state $\mathrm{A}$ in figure 10.

synchronous secondary flows. The experimental characterizations at $R e_{a}=240$ of $\mathrm{A}$ at $\omega=1.0$ and $\mathrm{B}$ at $\omega=6.1$ are shown as space-time diagrams in figures $12(a)$ and $12(b)$. A and B can be clearly distinguished by the behaviour of the jets separating the Taylor cells. In figure $12(a)$ the jets of A shift half a wavelength every half-period, and in figure $12(b)$ the jets of $\mathrm{B}$ do not shift. The corresponding wavenumbers for these particular experimental runs are $k^{A}=3.0$ and $k^{B}=3.4$, consistent with the nonlinear computations.

Youd et al. (2003) noted that the most striking feature of A is the exponential growth and decay that the vortices experience every half-period. This behaviour is manifested in the computed kinetic-energy time series of $\mathrm{A}$ at $R e_{a}=240$ and $\omega=1$ shown in figure $12(c)$. At this low frequency, the response is quasi-steady to the continuous but slow change in $\operatorname{Re}(t)$. At $t=0$, corresponding to $\operatorname{Re}(t)=0$, the vortices are decaying exponentially and reach a minimum of kinetic-energy shortly after the motion of the cylinder becomes supercritical $\left(\operatorname{Re}(t)=\operatorname{Re}_{0, c}\right)$. The instantaneous $\operatorname{Re}(t)$ is shown in figure 12(e) (and in figure $12(f)$ for the $\omega=6.1$ case) as a solid line together with $\pm R e_{0, c}$ (dashed lines) so as to clearly identify the phases of the cycle during which the cylinder motion is super- and subcritical. The decay of the vortices can also be seen in the experimental space-time diagram of figure 12(a), where the vortices are observed to fade away (the intensity tends to an axially uniform shade of grey). Subsequently, the vortices grow exponentially until the flow saturates nonlinearly at $t / T=0.133$ and algebraic growth follows. The algebraic growth continues to $t / T=0.25$, when the maximum amplitude of the modulations $\operatorname{Re}(t)=240$ is achieved, and it is followed by algebraic decay as the cylinder slows down. In the experiments, this long phase of algebraic behaviour is distinguished by a greater contrast in the reflected light across the vortices. When the rotation of the cylinder again becomes subcritical, the vortices decay exponentially. Owing to the spatio-temporal symmetry $S^{\prime}$, this sequence is repeated every half-period, i.e. the kinetic-energy time series is $T / 2$ periodic.

The behaviour of B at a higher frequency $\omega=6.1$ is qualitatively different. In this case, the flow does not have time to adjust quasi-steadily to the rapidly changing $\operatorname{Re}(t)$. This results in a delay of the response as can be seen in the kinetic-energy time series of figure $12(d)$, and also in the space-time diagram of figure $12(b)$. In 
(a)

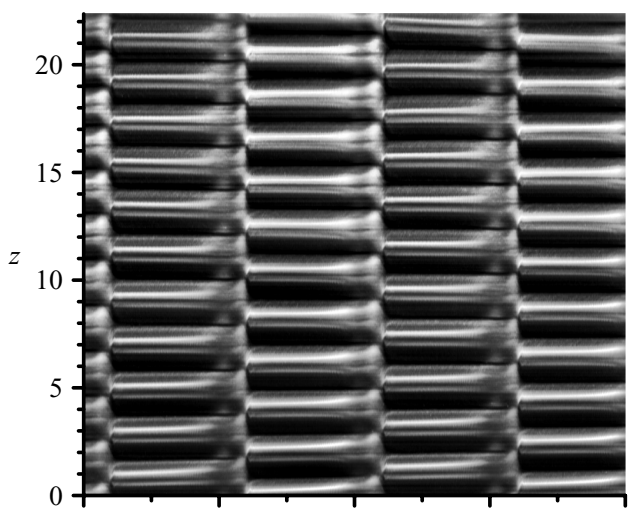

(c)

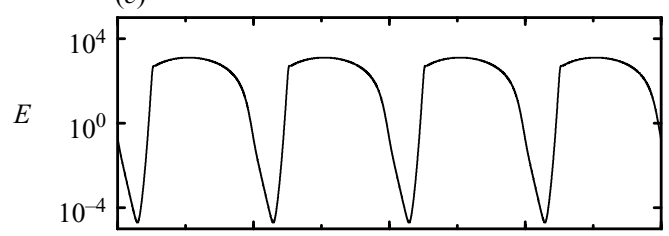

(e)

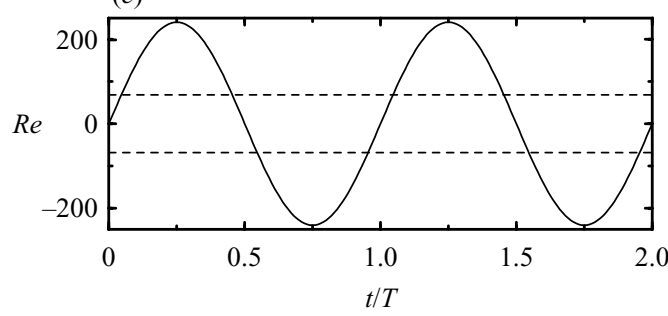

(b)

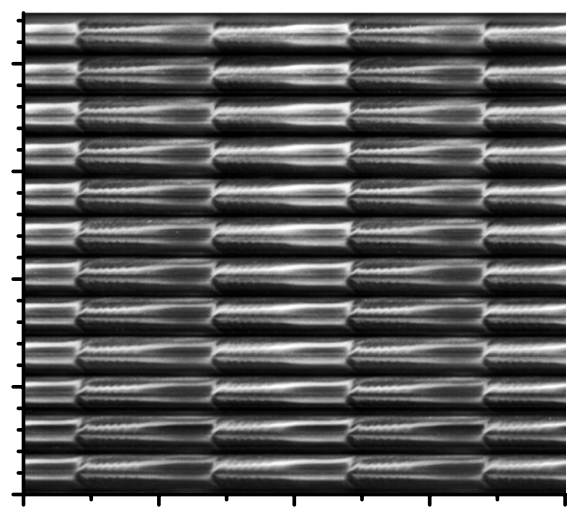

(d)

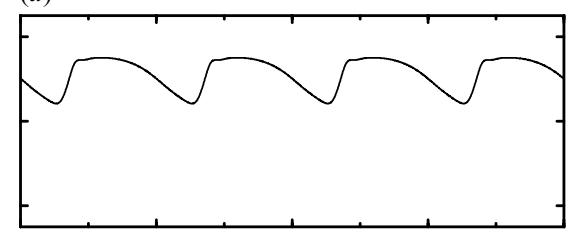

$(f)$

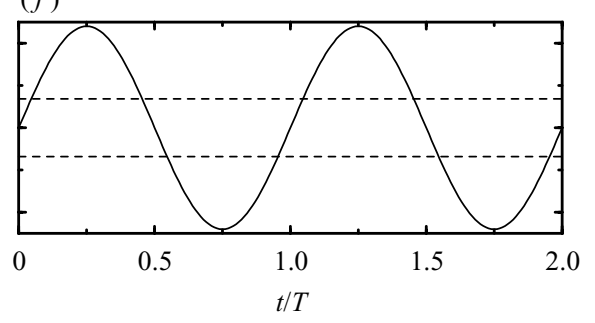

FIGURE 12. Experimental space-time diagrams of the time evolution at $R e_{a}=240$ of $(a)$ state $\mathrm{A}$ at $\omega=1$ with $k^{A}=3.0$ and $(b)$ state $\mathrm{B}$ at $\omega=6.1$ with $k^{B}=3.4$; the corresponding computed kinetic energies of the perturbation field $\boldsymbol{u}$ are shown in $(c)$ and $(d)$, and the corresponding instantaneous Reynolds numbers $R e(t)=R e_{a} \sin \omega t$ (solid curve) are given in $(e)$ and $(f)$ which also include $R e_{0, c}= \pm 68.19$ (dashed lines).

particular, the flow saturates nonlinearly at $t / T=0.215$, in comparison to $\mathrm{A}$ at $\omega=1$ which saturates at $t / T=0.133$. Although the flow is subcritical for the same fraction of the period in the two cases, since the period is six times shorter in viscous time units for $\mathrm{B}$ at $\omega=6.1$, and the growth rates for $\mathrm{A}$ and $\mathrm{B}$ in viscous time units are comparable $\left(\mathrm{e}^{-22.9 t}\right.$ and $\mathrm{e}^{-23.4 t}$, respectively), the exponential decay of $\mathrm{A}$ at $\omega=1$ during this fraction of time leads to vortices with minimum energy of about six orders of magnitude smaller than for $\mathrm{B}$ at $\omega=6.1$.

\subsection{Wavenumber selection of the flows}

The neutral stability curves shown in figure 7 indicate that at $R e_{a}=240$ solutions with a wide range of axial wavenumbers $k$ can be selected. The stability of these flows is limited by the Eckhaus bifurcation curves. In the experiments, the axial wavenumber that is selected depends on perturbations and initial conditions that are not completely controllable and cannot be fully characterized. Repeated experimental runs at the same point in parameter space $\left(R e_{a}, \omega\right)$ lead to states with different $k$ well within the Eckhaus-stable band. Similar multiplicity of states in classical Taylor-Couette flow is 


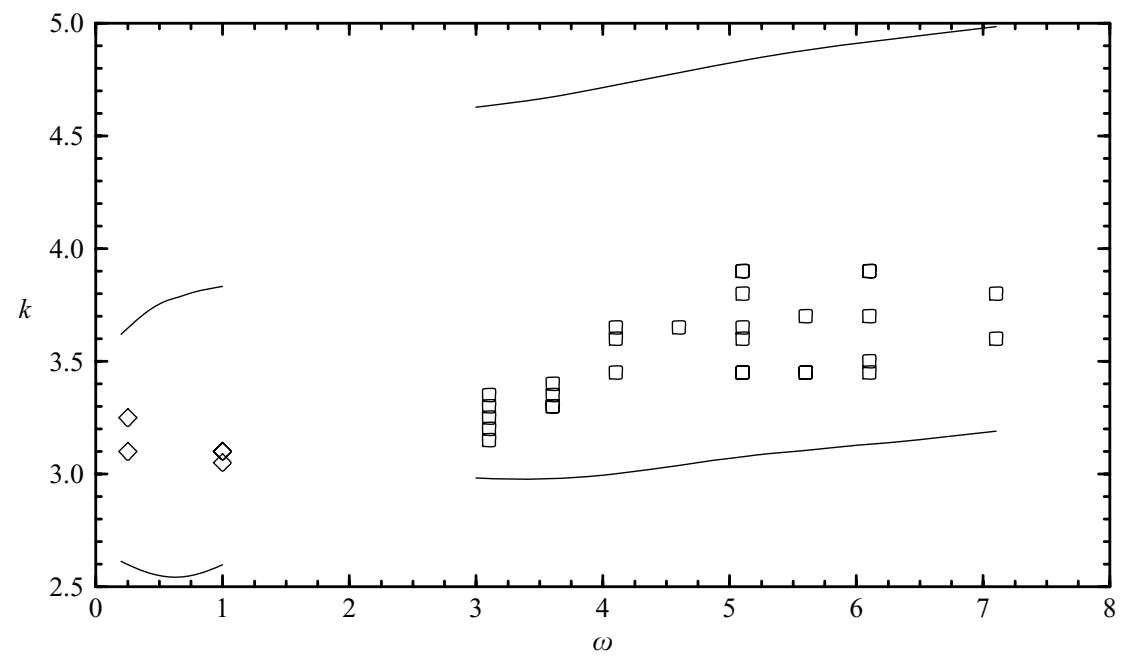

FiguRE 13. Experimentally observed axial wavenumbers $k$ of $A(\diamond)$ and $\mathrm{B}(\square)$ in several experimental runs at $R e_{a}=240$. The solid lines correspond to the Eckhaus curves from the nonlinear computations.

well-known (Coles 1965). Figure 13 shows the axial wavenumbers of A (diamonds) and B (squares) observed in several experimental runs at $R e_{a}=240$ and various $\omega$. (Results in the interval $1<\omega<3$ are presented in $\S 5$ as they correspond to the competition region.) After transients have decayed, the Taylor cells are of uniform wavelength and no defects are observed. All the observed $k$ are inside the Eckhaus bands as determined by nonlinear computations, shown in solid lines in figure 13 .

\section{Mode competition: bifurcation scenario}

We have numerically investigated the competition between A and B flow states in a wide region of parameter space around the codimension-two point where they bifurcate simultaneously, $\left(\omega, R_{a, c}\right)=(3.936,119.4)$. The critical axial wavenumbers for $\mathrm{A}$ and $\mathrm{B}$ from Floquet analysis are $k_{c}^{A}=2.71$ and $k_{c}^{B}=3.82$. Let us assume for now that only one $\mathrm{A}$ and one $\mathrm{B}$, with fixed incommensurate $k_{c}^{A}$ and $k_{c}^{B}$, exist in the neighbourhood of this codimension-two point. This classical simplification allows one to interpret the bifurcation scenario in terms of standard equivariant bifurcation theory while preserving the essentials of the competition dynamics. The effects of the presence of two families of flows A and B, given by the Eckhaus bands, will be addressed in the next paragraph. Figure 14 shows the bifurcation diagram of the competition between $\mathrm{A}$ and $\mathrm{B}$ in a wide region of parameter space. For $R e_{a}$ and $\omega$ in region 1 the basic state $\mathrm{mC}$ is the only flow that exists and it is stable. Crossing into region $2, \mathrm{mC}$ becomes unstable and $\mathrm{A}$ emerges as a stable state via a synchronous symmetry-breaking bifurcation. Upon crossing into region 3, B emerges from another synchronous bifurcation from $\mathrm{mC}$, but it is unstable and only stabilizes in region 4 where an unstable mixed-mode $\mathrm{AB}$ is born. In region 4 , A and B coexist and are both stable. Depending on initial conditions, either of them may be obtained. The situation is analogous when starting from region 1 and crossing into regions 6 and 5 to reach region 4 . As all the bifurcations are supercritical, these paths in parameter space can be reversed and the same results hold. The detailed analysis of this bifurcation is presented in the Appendix in terms of equivariant normal-form theory. Figure 15 


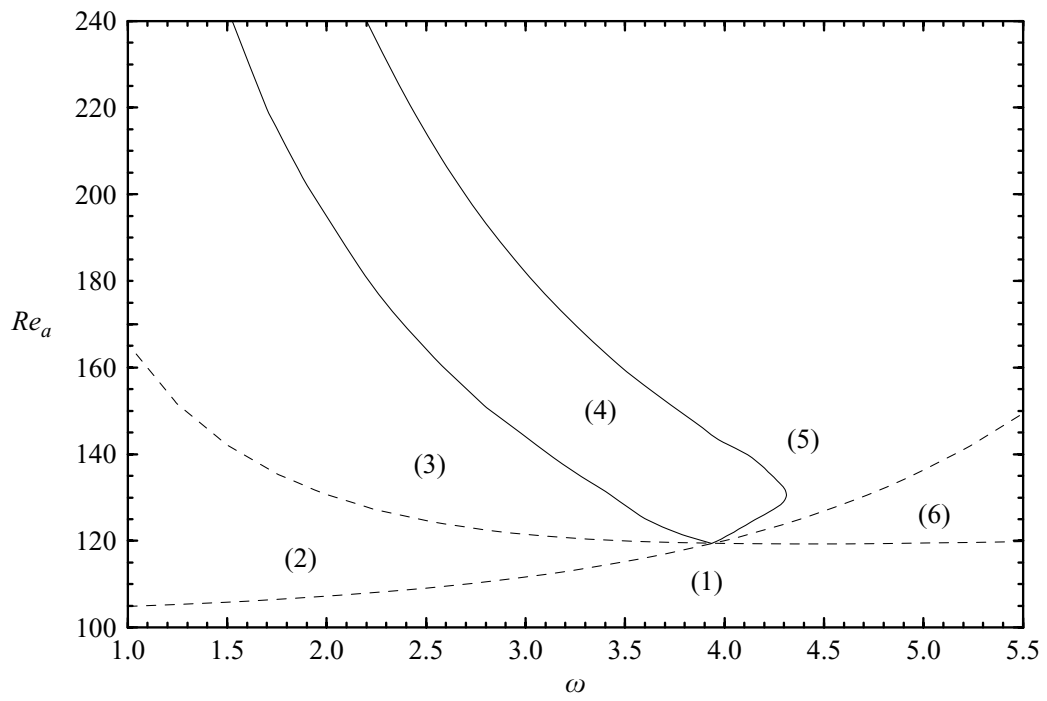

Figure 14. Bifurcation diagram of the competition between types A (reversing) and B (non-reversing) Taylor vortex flows. Bracketed numbers correspond to regions in parameter space with qualitatively different dynamics, described in the text. A schematic of their phase portraits is shown in figure 15 .
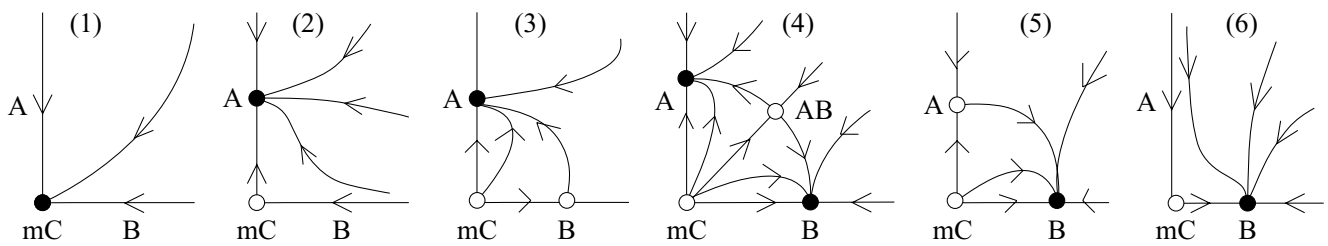

FIGURE 15. Schematic of the phase portraits of the mode competition dynamics in the six regions delimited by the bifurcation curves in figure 14. Open (closed) circles are unstable (stable) fixed points of the Poincaré map $\mathscr{P}$.

shows a schematic of the phase portraits in the six regions of parameter space, indicating the stability of the flows, summarizing the results in the Appendix.

Note that at any point in parameter space with $R e_{a}>R e_{a, c}$ there exist not a single A or a single B, but two families of these flows. Therefore, on performing nonlinear computations to determine the bifurcation curves in figure 14 , it is necessary to consider $\mathrm{A}$ and $\mathrm{B}$ with different axial wavenumbers (all of them multiples of the small fundamental $k_{f}=0.15$ ). Their stability is determined as explained in $\S 2.1$. Say we start a simulation with $\omega=3.2$ and $R e_{a}=140$ and obtain $\mathrm{B}_{50}$ with wavenumber $k^{B}=3.75$, corresponding to 50 Taylor cells $(l=25)$. Upon decreasing the Reynolds number to $R e_{a}=138$, we cross from region 4 to region $3, \mathrm{~B}_{50}$ becomes unstable and the system evolves to $\mathrm{A}_{38}$ (with $k^{A}=2.85$ ). However, at this $R e_{a}$ and $\omega, \mathrm{B}_{52}$ (with $\left.k^{B}=3.9\right)$ is still stable and is only destabilized when $R e_{a}$ is further reduced to 137 . Likewise, for each pair $\left(A_{2 l}, B_{2 l^{\prime}}\right)$ there exists a set of bifurcation curves. Region 4 in figure 14 is defined as all points in parameter space where at least a stable $A$ and a stable B coexist simultaneously. The boundaries of region 4 consist of segments from the bifurcation curves at which various A and B lose stability. Note that in region 4, there exists not a single unstable mixed mode AB, but a family of such modes. 


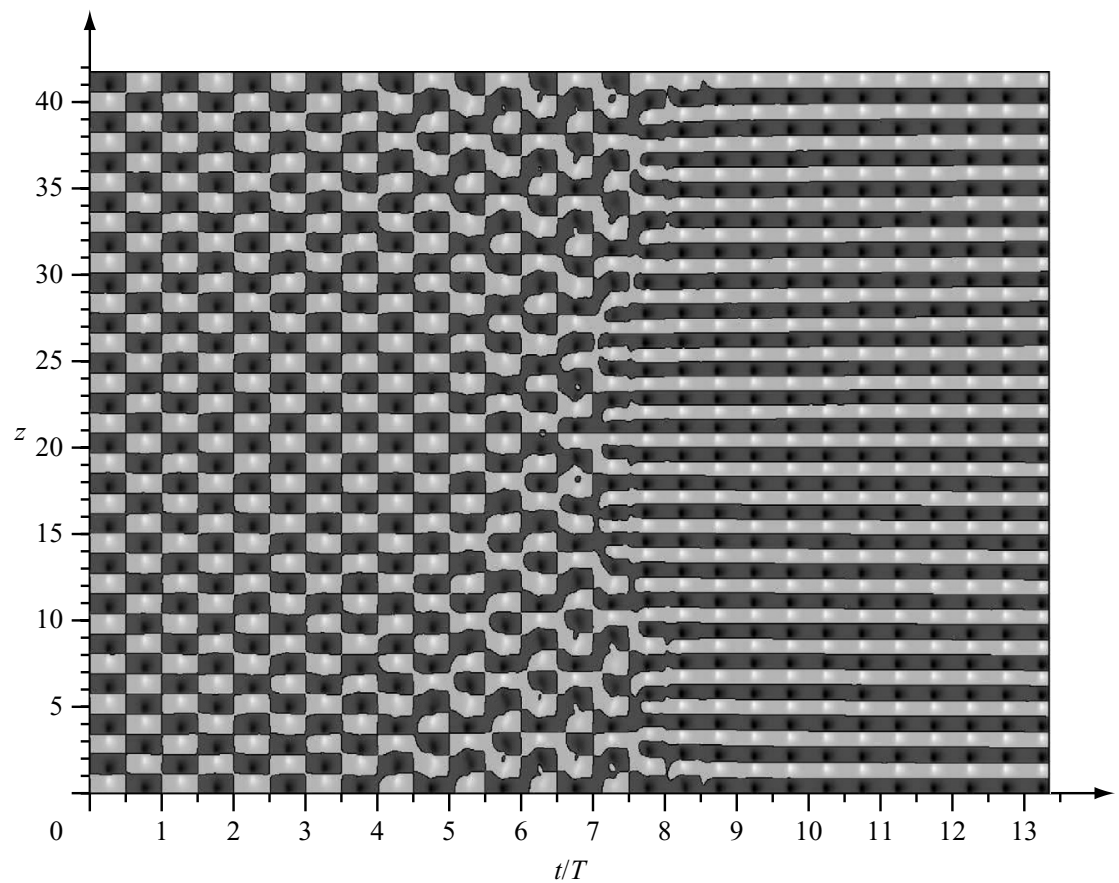

FIGURE 16. Space-time diagram showing an azimuthal vorticity colour-map at $(r, \theta)=\left(r_{o}, 0\right)$ over time and the whole computational domain in the axial direction $z \in[0, \Lambda]$. Black (grey) corresponds to positive (negative) values. The initial condition is $\mathrm{A}_{36}$ at $\omega=4$ and $R e_{a}=140$. At $t=0$ the frequency is increased to $\omega=4.2$ and the system starts evolving to $\mathrm{B}_{48}$.

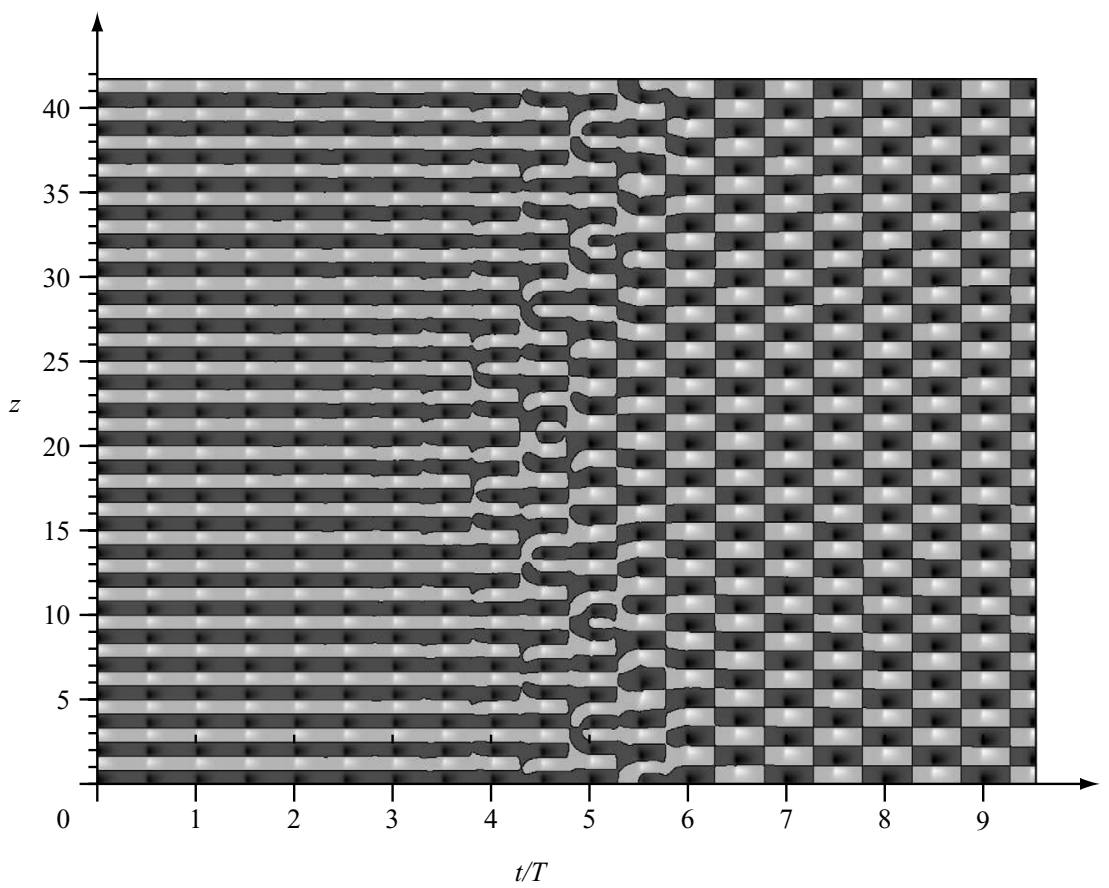

FIGURE 17. As figure 16, but the initial condition is $\mathrm{B}_{50}$ at $\omega=3.2$ and $R e_{a}=140$. At $t=0$ the frequency is decreased to $\omega=3.0$ and the system evolves to $\mathrm{A}_{38}$. 
A typical example of the nonlinear dynamics of the transition from A to B when crossing from region 4 to region 5 is shown in the space-time diagram of figure 16 . In the plot, we have arbitrarily set $t=0$; however by this time the flow had evolved for many periods until a stable $\mathrm{A}_{36}$ was obtained at $R e_{a}=140$ and $\omega=4$ (region 4). Then, at $t=0$ a small perturbation (with energy several orders of magnitude lower than that of the flow) was introduced and the frequency was raised to $\omega=4.2$, thus entering region 5 where $\mathrm{A}$ is unstable. After a transient (of about 10 periods) during which $\mathrm{A}$ and $\mathrm{B}$ compete, the flow settles to $\mathrm{B}_{48}$. Before the transition, the halfwavelength axial shifts of $\mathrm{A}_{36}$ every half-period are evident. Following the transition to $\mathrm{B}_{48}$, the flow no longer shifts axially and has a larger wavenumber. Figure 17 shows the transition from $\mathrm{B}$ to $\mathrm{A}$ when crossing from region 4 to region 3 . The initial state is $\mathrm{B}_{50}$ at $R e_{a}=140$ and $\omega=3.2$, and upon decreasing the frequency to $\omega=3.0$, the flow evolves to $\mathrm{A}_{38}$.

\subsection{Experimental dynamics of the competition at $R e_{a}=240$}

We have conducted experiments at $R e_{a}=240$ to verify the bifurcation scenario and identify the regions in parameter space predicted by the nonlinear computations. In the low-frequency regime, $\omega \leqslant 1$, corresponding to region 3 in figure 14, the only observed flow is A. For frequencies $\omega \geqslant 3$, corresponding to region 5, only B is observed. These flows are characterized by a well-defined time-independent axial wavenumber $k$. The observed values of $k$ are given in figure 13 .

The experimental procedure to detect the boundaries of the coexistence region 4 is as follows. The apparatus is operated at $\omega \geqslant 6$ in region 5 and far from region 4 as determined by the nonlinear computations, which at $R e_{a}=240$ spans $\omega \in(1.53,2.20)$. After transients have decayed, a spatially regular B state is obtained. Then, the frequency is decreased to say $\omega=5$. There is a rapid initial adjustment via vortex mergers or splits (defects in the pattern which appear at random axial locations) on a time scale of about $2 \pi / \omega$ (on the order of $d^{2} / v$ for the range of frequencies investigated). This is followed by a slower adjustment of the wavelength on the viscous time scale associated with the distance between endwalls $\left(\Gamma d^{2} / \nu\right)$. The frequency is subsequently reduced further and different $\mathrm{B}$ are obtained at various $\omega$. Following each of these changes in $\omega$, we observe the same type of rapid adjustment via defects followed by the gradual relaxation. However, close to region 4 (at about $\omega \sim 3$ ) the defects do not vanish after a period but are persistent in time. Figure 18 shows a typical space-time diagram of an experimental run in this parameter regime, with $R e_{a}=240$ and $\omega=2$. The flow does not settle on a particular axial wavenumber and there are abrupt appearances of defects over the whole experimental run (which lasted 36 periods, corresponding to about 120 viscous times). The defects are evident when the flow saturates nonlinearly, and when $\operatorname{Re}(t)$ peaks at about $|\operatorname{Re}(t)|=240$ the cells are more spatially uniform. The defects appear at random axial locations each half-period and the dominant axial wavenumber is apparently randomly selected in time with values $k \in[2.9,4.0]$, showing no clear trend (a few close-ups of the images in figure 18 are presented in figure 19 showing typical defects). The flow cannot be regarded as either $\mathrm{A}$ or $\mathrm{B}$; the jets separating the vortices shift by irregular amounts in space and time. This type of behaviour is observed following decreases in $\omega$, until we reach $\omega=1$. Following a decrease to $\omega=1$, the defects quickly disappear within about one period, and the Taylor cells adjust slowly to a uniform axial wavenumber $k$. At this $\omega$, the jets shift by half a wavelength every half-period, corresponding to A. This whole scenario is reversed with increasing $\omega$, i.e. we did not detect any hysteresis with varying $\omega$. 

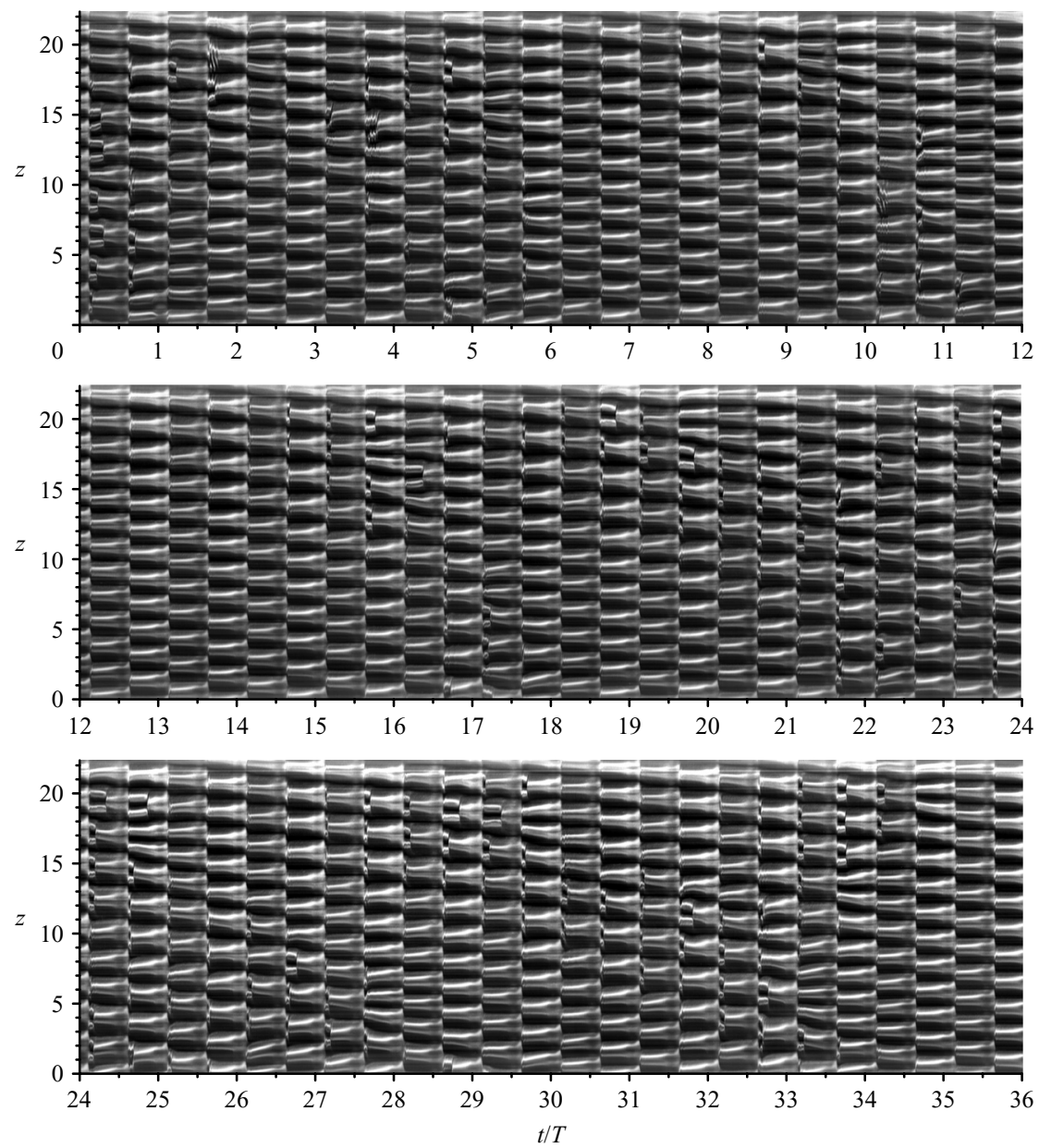

FIGURE 18. Space-time diagram showing the time evolution of a noise-sustained flow in region 4 at $\omega=2.0$ and $R e_{a}=240$ over 36 periods, corresponding to 113 viscous time units. At $t / T=0$, the frequency was changed from a stable flow at $\omega=1.0$.
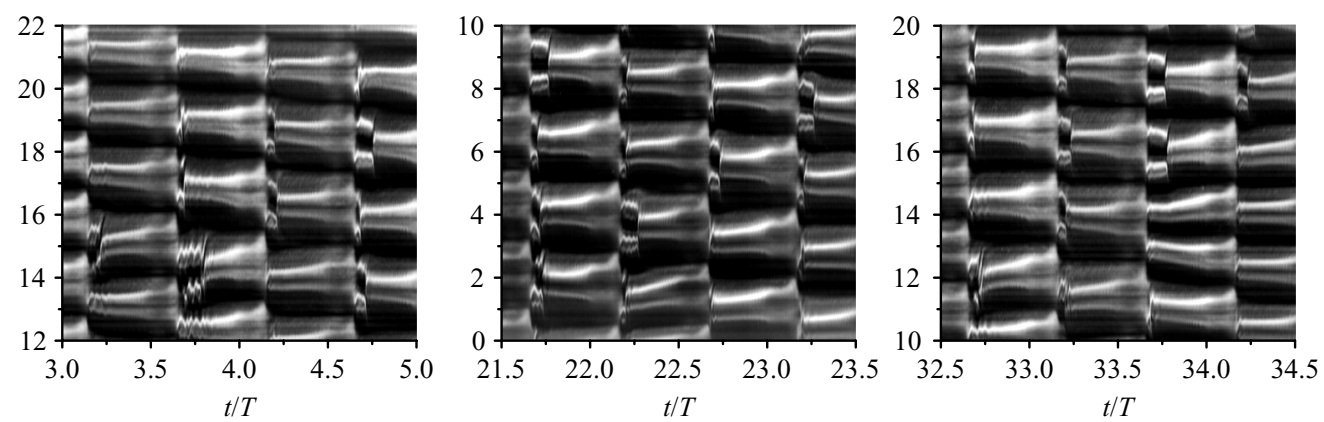

FIGURE 19. Close-ups of the images in figure 18 showing typical defects.

\section{Discussion and conclusions}

Nonlinear dynamics of the time-modulated Taylor-Couette system has been investigated experimentally and numerically by solving the unsteady three- 
dimensional Navier-Stokes equations. The aspect ratio of the experimental apparatus is $\Gamma=115$, whereas in the nonlinear computations a long periodic annulus of wavelength $\Lambda=41.89$ has been considered. The study focused on temporal modulations about a zero mean. In this case, the basic state is characterized by the presence of a $Z_{2}$ spatio-temporal symmetry, $S$, in addition to the spatial symmetries of the classical Taylor-Couette system.

The onset of instability has been determined by Floquet analysis of the basic state, which shows that two distinct Taylor vortex flows A (reversing) and B (non-reversing) are born at synchronous symmetry-breaking bifurcations of the modulated Couette flow. This result resolves the discrepancies between previous Floquet analyses for finite gaps (Carmi \& Tustaniwskyj 1981; Barenghi \& Jones 1989) and narrow gaps (Riley \& Laurence 1976). Moreover, experimental evidence of the existence of flows A and $\mathrm{B}$ has been provided for the first time. The dynamical behaviour of $\mathrm{A}$ and $\mathrm{B}$ and the observed axial wavenumbers are in good agreement with the predicted values from Floquet analysis and nonlinear computations. The stability of the basic state has been investigated using the half-period-flip map $\mathscr{H}$ instead of the Poincare map $\mathscr{P}=\mathscr{H}^{2}$. This approach sheds light on the nature of the symmetry-breaking bifurcations to $\mathrm{A}$ and $\mathrm{B}$, while also having the practical advantage of halving the computational time. The bifurcation to B preserves the spatio-temporal symmetry $S$, whereas the bifurcation to A breaks it and a new spatio-temporal symmetry $S^{\prime}$ emerges, consisting of $S$ composed with an axial shift of half a wavelength. Flows with precisely the same symmetries as A and B arise in other systems, such as the periodically driven cavity flow (Marques et al. 2004) and cylinder wake flows (Barkley \& Henderson 1996), via analogous bifurcations in the transition from two-dimensional to three-dimensional flow (Blackburn, Marques \& Lopez 2005). However, in modulated Couette flow, the transition is from one-dimensional to two-dimensional flow, highlighting that the symmetry group of the system, and in particular of the flow from which the bifurcations occur, determines the spatio-temporal characteristics of the bifurcated flows regardless of the specifics of the problem. Therefore, a careful consideration of the symmetries of the system not only provides the key to understanding the nature of the two bifurcated flows, but furnishes the setting to compare systems with inherently different physical instability mechanisms.

In previous studies, direct numerical simulation had been focused on investigating the codimension-one bifurcations leading to the two states with distinct spatiotemporal symmetries. The flow in the wake of a cylinder is governed by a single parameter, the Reynolds number $R e$, so that a study of the mode competition between A and B is impractical (two parameter variations are required to explore the competition dynamics organized by the codimension-two point where both modes bifurcate simultaneously). State A bifurcates first at lower Reynolds number, so a pure state $\mathrm{B}$ is never observed in the cylinder wake experiments and can only be obtained in the computations by prescribing the wavenumber. The transition from $\mathrm{A}$ to $\mathrm{B}$ is found to be gradual in experiments (Williamson 1996) and to some extent in nonlinear computations (Henderson 1997). Barkley, Tuckerman \& Golubitsky (2000) proposed a system of two coupled amplitude equations based on the assumption of the existence of a codimension-two point where A and B bifurcate simultaneously; the experimentally observed transition was described as a one-dimensional path (parameterized with $R e$ ) in a two-parameter model. In modulated Taylor-Couette flow, the study of the competition between the analogous states A and B is more straight-forward as the two parameters, $R e_{a}$ and $\omega$, can be varied. Youd et al. (2003) obtained A and B with nonlinear computations for the first time. In a subsequent study, Youd et al. (2005) 
also investigated the secondary transition to three-dimensional flows and showed that non-reversing spiral flows occur. However, their approach, in which they computed a single mode with a prescribed wavelength, was unable to study mode competition and pattern selection. At about the same time, Youd \& Barenghi (2005) also considered the problem in a finite annulus with stationary rigid endwalls and relatively short aspect ratios, and found that both states A and B continue to exist. Although mode competition could have been studied along the lines presented here, they did not perform such an analysis and only considered two modulation frequencies, $\omega=3$ and $\omega=7$. The effects of rigid endwalls and finite aspect ratios on the competition between states A and B remains an open question.

In this paper we have numerically and experimentally investigated the competition between $\mathrm{A}$ and $\mathrm{B}$ in the two-dimensional parameter space $\left(\omega, R e_{a}\right)$. In the nonlinear computations, $\mathrm{A}$ and $\mathrm{B}$ coexist and the two are stable in a narrow region of parameter space where a mixed mode $A B$ also exists but is unstable. In fact, in this region there are families of $\mathrm{A}$ and $\mathrm{B}$ and mixed modes. In the experiments neither $\mathrm{A}$ nor $\mathrm{B}$ has been observed in the coexistence region. Instead, we have found that noise sustains flows with irregular time-dependent axial wavenumber. Outside this region, for lower frequency only A is observed, and for higher frequency only B is observed, in good agreement with the numerical simulations and the Floquet stability analysis. This finding poses a fundamental question of interest in mode competition and pattern formation. In particular it is noteworthy that the wavenumber selection fails only in the mid-frequency coexistence region. For lower frequencies, where noise effects are expected to be more pronounced due to the long time that perturbations have to grow and decay, regular spatially and temporally periodic flows were in fact observed. It is thus of interest to determine the nature of the flows observed in the coexistence region, and in particular to determine if it is noise sustaining a mixed mode generated by a combination of $\mathrm{A}$ and $\mathrm{B}$ with a wide range of axial wavenumbers.

This work was supported by the National Science Foundation grant DMS05052705, the Spanish Ministry of Education and Science grants FIS2004-01336 and AP-2004-2235, and Catalonian Government grant SGR-00024. Part of the work was done during M. Avila's visit to the Department of Mathematics and Statistics, Arizona State University, whose kind hospitality is warmly appreciated. Computational resources of ASU's Fulton HPCI are greatly appreciated.

\section{Appendix. Normal-form analysis of the double pitchfork-of-revolution bifurcation}

The dynamics of the problem in the neighbourhood the codimension-two point, where the periodic base state bifurcates simultaneously to modes A and B, is axisymmetric. The symmetry group of the problem reduces to $\mathscr{G}=Z_{2}^{S T} \times O(2)$, where $Z_{2}$ is generated by the space-time symmetry $S$. The possible bifurcations and corresponding normal forms in systems with this symmetry were obtained in Marques et al. (2004), where the codimension-one bifurcations were analysed in detail. Here, we start with the normal form and analyse the dynamics associated with the codimension-two bifurcation. Since mode A breaks the space-time symmetry $S$, and mode B preserves it, the normal form for the half-period-flip map $\mathscr{H}$, up to third 
order, is

$$
\mathscr{H}:\left\{\begin{array}{l}
A \mapsto A\left(-1+\mu+a|A|^{2}+b|B|^{2}\right) \\
B \mapsto B\left(+1+v+c|A|^{2}+d|B|^{2}\right)
\end{array}\right.
$$

where $A$ and $B$ are the complex amplitudes corresponding to the bifurcating modes $\mathrm{A}$ and $\mathrm{B}$ respectively. Since all the coefficients $(\mu, v, a, b, c, d)$ are real, the dynamics of the phases of $A$ and $B$ are trivial; writing $A$ and $B$ in terms of their moduli and phases, $A=r_{1} \mathrm{e}^{\mathrm{i} \phi_{1}}$ and $B=r_{2} \mathrm{e}^{\mathrm{i} \phi_{2}}$, we obtain

$$
\mathscr{H}:\left\{\begin{array} { l } 
{ r _ { 1 } \mapsto r _ { 1 } ( - 1 + \mu + a r _ { 1 } ^ { 2 } + b r _ { 2 } ^ { 2 } ) } \\
{ r _ { 2 } \mapsto r _ { 2 } ( + 1 + v + c r _ { 1 } ^ { 2 } + d r _ { 2 } ^ { 2 } ) , }
\end{array} \quad \left\{\begin{array}{l}
\phi_{1} \mapsto \phi_{1} \\
\phi_{2} \mapsto \phi_{2},
\end{array}\right.\right.
$$

and the phases $\phi_{1}$ and $\phi_{2}$ remain constant. Assuming that $a$ and $d$ are not zero (they are negative in our problem), re-scaling $r_{1}$ and $r_{2}$ gives

$$
\mathscr{H}:\left\{\begin{array}{l}
r_{1} \mapsto r_{1}\left(-1+\mu-r_{1}^{2}-\zeta r_{2}^{2}\right) \\
r_{2} \mapsto r_{2}\left(+1+v-\delta r_{1}^{2}-r_{2}^{2}\right),
\end{array}\right.
$$

with two unfolding parameters $\mu$ and $\nu$, and two constants $\zeta$ and $\delta$ whose exact values depend on the problem considered. In the neighbourhood of the codimension-two point the two bifurcation parameters $\mu$ and $v$ are linearly related to $R e_{a}$ and $\omega$ by

$$
\left.\begin{array}{l}
\mu=R e_{a}-10.56 \omega-77.86, \\
\nu=R e_{a}+0.8992 \omega-122.96
\end{array}\right\}
$$

and the two constants are $\zeta=1.9670$ and $\delta=1.9022$. These values have been obtained from the numerical data in figure 14 . The action of $\mathscr{G}_{s}=O(2) \times Z_{2}$ leaves the amplitudes $r_{1}$ and $r_{2}$ invariant, but acts non-trivially on the phases (Marques et al. 2004):

$$
\left.\begin{array}{l}
T_{a}\left(\phi_{1}, \phi_{2}\right)=\left(\phi_{1}+k_{1} a, \phi_{2}+k_{2} a\right), \\
K_{z}\left(\phi_{1}, \phi_{2}\right)=\left(-\phi_{1},-\phi_{2}\right), \\
H\left(\phi_{1}, \phi_{2}\right)=\left(\phi_{1}+\pi, \phi_{2}\right),
\end{array}\right\}
$$

where $k_{1}$ and $k_{2}$ are the critical axial wavenumbers of modes $\mathrm{A}$ and $\mathrm{B}$ respectively. These actions are easy to obtain by considering that the bifurcating eigenvectors are of the form $A \mathrm{e}^{\mathrm{i} k z} \boldsymbol{v}(r, t)$ because the base state $\mathrm{mC}$ is independent of $(\theta, z)$. We assume that $k_{1} / k_{2}$ is irrational (non-resonant case), i.e. the critical wavenumbers of the two modes are not in a rational ratio, as is the case in our problem.

In order to describe the dynamics associated with the normal form (A.3), it is convenient to obtain the fixed points of the Poincare map $\mathscr{P}=\mathscr{H}^{2}$. Up to third order,

$$
\mathscr{P}:\left\{\begin{array}{l}
r_{1} \mapsto r_{1}\left(1+2 \mu-2 r_{1}^{2}-2 \zeta r_{2}^{2}\right) \\
r_{2} \mapsto r_{2}\left(1+2 \nu-2 \delta r_{1}^{2}-2 r_{2}^{2}\right)
\end{array}\right.
$$

The Poincaré map may have up to four different fixed points, depending on the values of $\zeta$ and $\delta$, and on the region in parameter space $(\mu, \nu)$ considered. The fixed points 
in phase space $\left(r_{1}, r_{2}\right)$ are

$$
\mathrm{mC}=(0,0), \quad \mathrm{A}=(\sqrt{\mu}, 0), \quad \mathrm{B}=(0, \sqrt{v}), \quad \mathrm{AB}=\left(\sqrt{\frac{\zeta v-\mu}{\zeta \delta-1}}, \sqrt{\frac{\delta \mu-v}{\zeta \delta-1}}\right)
$$

and they have been labelled according to the solutions of the present problem.

The modulus dynamics (A.6) are identical to the modulus dynamics of the doubleHopf bifurcation (dH), and the results in Kuznetsov (2004) apply to the present case. In particular, the bifurcation scenario of figure 14 is the analogue of type I of the 'simple' case of $\mathrm{dH}$, for which $\zeta>0, \delta>0$ and $\theta \delta>1$ (Kuznetsov 2004). However, the interpretation of the dynamics associated with the fixed points (A.7) changes. The Hopf bifurcations in $\mathrm{dB}$ become pitchfork-of-revolution bifurcations (since the phases remain constant). $\mathrm{A}$ and $\mathrm{B}$ are limit cycles in $\mathrm{dH}$, and here they are fixed points; and $\mathrm{AB}$, a quasi-periodic solution in $\mathrm{dH}$, is also a fixed point here. As the phase dynamics here refers to translations in $z$ while in the $\mathrm{dH}$ it is associated with time, $\mathrm{A}$ and $\mathrm{B}$ are periodic solutions in $z$, while $\mathrm{AB}$ is quasi-periodic in space. Finally, in $\mathrm{dH}$ one is dealing with an ODE system, so the curves in the phase portraits are orbits in $\left(r_{1}, r_{2}\right)$ phase space; here we are dealing with maps $(\mathscr{P}$ and $\mathscr{H})$ and so the curves in the phase portraits are invariant manifolds, containing an infinite number of discrete orbits.

\section{REFERENCES}

Avila, M., Meseguer, A. \& Marques, F. 2006 Double Hopf bifurcation in corotating spiral Poiseuille flow. Phys. Fluids 18, 064101.

BARENGHi, C. F. \& Jones, C. A. 1989 Modulated Taylor-Couette flow. J. Fluid Mech. 208, 127-160.

BARKLey, D. \& Henderson, R. D. 1996 Three-dimensional Floquet stability analysis of the wake of a circular cylinder. J. Fluid Mech. 322, 215-241.

Barkley, D., Tuckerman, L. S. \& Golubitsky, M. 2000 Bifurcation theory for three-dimensional flow in the wake of a circular cylinder. Phys. Rev. E 61, 5247-5252.

BelisLe, M. J. 2007 Experiments on mode competition in temporally-modulated Taylor-Couette flow. Master's thesis, Arizona State University, Tempe, Arizona.

Blackburn, H. M., Marques, F. \& Lopez, J. M. 2005 Symmetry breaking of two-dimensional time-periodic wakes. J. Fluid Mech. 522, 395-411.

Carmi, S. \& Tustaniwskyj, J. I. 1981 Stability of modulated finite-gap cylindrical Couette flow: Linear theory. J. Fluid Mech. 108, 19-42.

Coles, D. 1965 Transition in circular Couette flow. J. Fluid Mech. 21, 385-425.

Dominguez-Lerma, M. A., Cannell, D. S. \& Ahlers, G. 1986 Eckhaus boundary and wave-number selection in rotating Couette-Taylor flow. Phys. Rev. A 34, 4956-4970.

Donnelly, R. J. 1964 Experiments on the stability of viscous flow between rotating cylinders. III. Enhancement of stability by modulation. Proc. R. Soc. Lond. A 281, 130-139.

Gauthier, G., Gondret, P. \& Rabaud, M. 1998 Motions of anisotropic particles: Application to visualization of three-dimensional flows. Phys. Fluids 10, 2147-2154.

HaLl, P. 1975 The stability of unsteady cylinder flows. J. Fluid Mech. 67, 29-63.

HALl, P. 1983 On the nonlinear stability of slowly varying time-dependent viscous flows. J.Fluid Mech. 126, 357-368.

Henderson, R. D. 1997 Nonlinear dynamics and pattern formation in turbulent wake transition. J. Fluid Mech. 352, 65-112.

KuZnetsov, Y. A. 2004 Elements of Applied Bifurcation Theory, 3rd edn. Springer.

LineK, M. \& Ahlers, G. 1998 Boundary limitation of wave numbers in Taylor-vortex flow. Phys. Rev. E 8, 3168-3174. 
Lopez, J. M. \& Marques, F. 2002 Modulated Taylor-Couette flow: Onset of spiral modes. Theore. Comput. Fluid Dyn. 16, 59-69.

Marques, F., Lopez, J. M. \& Blackburn, H. M. 2004 Bifurcations in systems with $Z_{2}$ spatiotemporal and $O(2)$ spatial symmetry. Physica D 189, 247-276.

Riecke, H. \& PaAP, H. G. 1986 Stability and wave-vector restriction of axisymmetric Taylor vortex flow. Phys. Rev. A 33, 547-553.

Riley, P. J. \& LAurence, R. L. 1976 Linear stability of modulated circular Couette flow. J. Fluid Mech. 75, 625-646.

SAVAş, Ö. 1985 On flow visualization using reflective flakes. J. Fluid Mech. 152, 235-248.

Swift, J. W. \& Wiesenfeld, K. 1984 Suppression of period doubling in symmetric systems. Phys. Rev. Lett. 52, 705-708.

TAkeuchi, D. I. \& JANkowski, D. F. 1981 A numerical and experimental investigation of the stability of spiral Poiseuille flow. J. Fluid Mech. 102, 101-126.

TAYLOR, G. I. 1923 Stability of a viscous liquid contained between two rotating cylinders. Phil. Trans. R. Soc. A 223, 289-343.

Tuckerman, L. S. \& Barkley, D. 1990 Bifurcation analysis of the Eckhaus instability. Physica D 46, 57-86.

Vogel, M., Hirsa, A. H. \& Lopez, J. M. 2003 Spatio-temporal dynamics of a periodically driven cavity flow. J. Fluid Mech. 478, 197-226.

Williamson, C. H. K. 1996 Vortex dynamics in the cylinder wake. Anu. Rev. Fluid Mech. 28, 477-539.

Youd, A. J. \& BARENGHI, C. F. 2005 Reversing and nonreversing modulated Taylor-Couette flow at finite aspect ratio. Phys. Rev. E 72, 056321.

Youd, A. J., Willis, A. P. \& Barenghi, C. F. 2003 Reversing and non-reversing modulated TaylorCouette flow. J. Fluid Mech. 487, 367-376.

Youd, A. J., Willis, A. P. \& Barenghi, C. F. 2005 Non-reversing modulated Taylor-Couette flows. Fluid Dyn. Res. 36, 61-73. 